\title{
CONTRIBUCIÓN AL CONOCIMIENTO DE LOS HEMERÓBIDOS DE PATAGONIA Y TIERRA DEL FUEGO (INSECTA, NEUROPTERA, HEMEROBIIDAE)
}

\author{
V. J. Monserrat*
}

\begin{abstract}
RESUMEN
Se aportan nuevos datos sobre la biología y distribución de 10 especies de hemeróbidos capturadas en 48 localidades de Patagonia y Tierra del Fuego. Se describen las larvas de Hemerobius bolivari Banks, 1910, Hemerobius chilensis Nakahara, 1965, Hemerobius stenopterus Monserrat, 1996, Sympherobius gayi Navás, 1910, Nomerobius cuspidatus Oswald, 1990 y Gayomyia falcata (Blanchard en Gay, 1851). Los estadios larvarios en los géneros Nomerobius y Gayomyia eran desconocidos hasta ahora.

Palabras clave: Neuroptera, Hemerobiidae, Neotropical, Patagonia, Tierra del Fuego, Argentina, Chile, Faunística, Larva, Planta substrato, Longevidad.
\end{abstract}

\section{ABSTRACT \\ Contribution to the Knowledge of the Brown-Lacewings from Patagonia and Tierra del Fuego (Insecta, Neuroptera, Hemerobiidae)}

New data on the biology and distribution of 10 species of brown-lacewings collected in 48 localities of Patagonia and Tierra del Fuego are given. Larvae of Hemerobius bolivari Banks, 1910, Hemerobius chilensis Nakahara, 1965, Hemerobius stenopterus Monserrat, 1996, Sympherobius gayi Navás, 1910, Nomerobius cuspidatus Oswald, 1990 and Gayomyia falcata (Blanchard in Gay, 1851) are described. Larval stages of genera Nomerobius and Gayomyia are described for the first time.

Key Words: Neuroptera, Hemerobiidae, Neotropical, Patagonia, Tierra del Fuego, Argentina, Chile, Faunistics, Larva, Plant substrate, Longevity.

\section{Introducción}

La familia Hemerobiidae incluye unas 600 especies y es una de las familias más interesantes dentro del orden Neuroptera debido a su amplia y casi cosmopolita distribución geográfica, al elevado número de ejemplares que con frecuencia constituyen sus poblaciones y especialmente por ser impor- tantes agentes de control biológico sobre pequeños fitófagos (McEwen et al., 2001).

La taxonomía y sistemática de esta familia ha sido recientemente tratada a nivel de género por Oswald (1993a) quien aporta valiosos datos que posibilitan una visión general muy detallada sobre la taxonomía, sistemática, filogenia y biogeografía de esta familia. Sin embargo aún queda mucho por

* Dept. de Zoología y Antropología Física, Universidad Complutense, E-28040 Madrid. E-mail: artmad@bio.ucm.es 
hacer ya que aún existen enormes áreas geográficas deficientemente muestreadas y aún quedan grandes lagunas sobre la morfología, variabilidad, taxonomía, biología, faunística y estadios juveniles de muchos taxones.

Referente a la fauna Neotropical, que venía estando bastante olvidada en relación a otras áreas biogeográficas, han sido recientemente publicados nuevos trabajos que han aportado nuevos datos que nos permiten un mayor conocimiento sobre la taxonomía, morfología, distribución y biología de numerosas especies de esta familia en Centro y Sudamérica. Se han publicado listados, nuevos taxones y la revisión de varios géneros de hemeróbidos perteneciente a la Fauna Neotropical, así como la de algún determinado país (Oswald, 1990, 1993b, 1994; González Olazo, 1992, 1993, 1996; Penny \& Lee, 1996; Monserrat, 1996, 1997, 1998, 2000; Monserrat \& Deretsky, 1999; Perez Gelabert \& Flint, 2001; Penny, 2002) que han contribuido a mejorar el conocimiento sobre la fauna de hemeróbidos de la Región Neotropical.

De estos trabajos se desprende la relativamente escasa la información que disponemos sobre la fauna de hemeróbidos de La Patagonia y de La Tierra del Fuego, ya que la mayoría de las citas y datos existentes se sitúan por encima del paralelo $35^{\circ} \mathrm{S}$, que existen pocos datos sobre la biología de la mayoría de las especies de esta región y que son muy amplias las zonas de las que no existe dato alguno.

Aumentar el nivel de conocimiento de los Hemerobiidae de esta enorme y particular extensión del cono sur sudamericano es el motivo de la presente contribución. En ella aportamos nuevos datos sobre la distribución geográfica, altitudinal, biología, morfología, estadios preimaginales y / o variabilidad de las 10 especies que se han encontrado durante los muestreos realizados.

\section{Material y métodos}

Se han tomado muestras en 48 localidades de Patagonia y Tierra del Fuego situadas al sur del paralelo $38^{\circ} \mathrm{S}$, tanto en la zona argentina como en la chilena, cuya ubicación administrativa, denominación, altitud sobre / bajo el nivel del mar, coordenadas geográficas y fechas de recolección se anotan numeradas en la lista adjunta:

\section{ARGENTINA}

1. PROVINCIA DE BUENOS AIRES, Tornquist, Cerro Tornquist, $400 \mathrm{~m}, 62^{\circ} 10^{\prime} \mathrm{W}, 38^{\circ} 04^{\prime} \mathrm{S}, 15$. XI. 2001.
2. PROVINCIA DE BUENOS AIRES, Tornquist, Parque Tornquist, Garganta Olvidada, $500 \mathrm{~m}, 62^{\circ} 03^{\prime} \mathrm{W}, 38^{\circ} 03^{\prime} \mathrm{S}$, 15. XI. 2001.

3. PROVINCIA DE BUENOS AIRES, Villarino, Laguna de Chasicó, Estancia La Aurora, entre -24 y $-45 \mathrm{~m}$, $62^{\circ} 55^{\prime} \mathrm{W}, 38^{\circ} 45^{\prime} \mathrm{S}, 22$. XI. 2001.

4. PROVINCIA DE BUENOS AIRES, Pehuen Có, 10 m, 613', 3900'S, 8. XII. 2001.

5. PROVINCIA DE BUENOS AIRES, Cabeza de Buey, 15 m, 62 $30^{\prime} \mathrm{W}, 39^{\circ} 10^{\prime} \mathrm{S}, 13$. XII. 2001.

6. PROVINCIA DE BUENOS AIRES, Mayor Buratovich, $15 \mathrm{~m}, 62^{\circ} 37^{\prime} \mathrm{W}, 39^{\circ} 15^{\prime} \mathrm{S}, 3$. XII. 2001.

7. PROVINCIA DE BUENOS AIRES, Carmen de Patagones, Ribera Río Negro, 10 m, 62 $49^{\circ}$ 'W, 40²4'S, 3. XII. 2001.

8. PROVINCIA DE BUENOS AIRES, Bahía Blanca, Arroyo Napostá, 20 m, 62 $10^{\prime} \mathrm{W}, 38^{\circ} 22^{\prime}$ S, 18. XII. 2001.

9. PROVINCIA DE BUENOS AIRES, Tornquist, Cerro Tornquist, $400 \mathrm{~m}, 62^{\circ} 10^{\prime} \mathrm{W}, 38^{\circ} 04^{\prime} \mathrm{S}, 18$. XII. 2001.

10. PROVINCIA DE BUENOS AIRES, Tornquist, Parque Tornquist, Garganta Olvidada, $500 \mathrm{~m}, 62^{\circ} 03^{\prime} \mathrm{W}, 38^{\circ} 03^{\prime} \mathrm{S}$, 18. XII. 2001.

11. PROVINCIA DE BUENOS AIRES, Médanos, Villarino, 25 m, 62 ${ }^{\circ} 54^{\prime} \mathrm{W}, 38^{\circ} 46^{\prime} \mathrm{S}, 19$. XII. 2001.

12. PROVINCIA DE BUENOS AIRES, Villarino, Laguna de Chasicó, Estancia La Aurora, entre - 24 y - 45 m, $62^{\circ} 55^{\prime} \mathrm{W}, 38^{\circ} 45^{\prime} \mathrm{S}, 19$. XII. 2001.

13. PROVINCIA DE SANTA CRUZ, El Calafate, Punta Bandera, 190 m, 72 $47^{\prime} \mathrm{W}, 50^{\circ} 68^{\prime}$ 'S, 5. I. 2002.

CHILE

14. XII REgIÓN, MAGALlANES, Punta Arenas, Reserva Forestal Laguna Parrillar, $80 \mathrm{~m}, 7^{\circ} 15^{\prime} \mathrm{W}, 53^{\circ} 24^{\prime} \mathrm{S}, 10$. I. 2002.

15. XII REGIÓN, MAGALlANES, Lago Blanco, $500 \mathrm{~m}$, $68^{\circ} 57^{\prime} \mathrm{W}, 5^{\circ} 01^{\prime} \mathrm{S}, 11$. I. 2002.

\section{ARGENTINA}

16. PROVINCIA DE TIERRA DEL FUEGO, Estancia Despedida, $120 \mathrm{~m}, 68^{\circ} 15^{\prime} \mathrm{W}, 53^{\circ} 58^{\prime} \mathrm{S}$, 12. I. 2002.

17. PROVINCIA DE TIERRA DEL FUEGO, Tolhuin, $250 \mathrm{~m}$, $67^{\circ} 13^{\prime} \mathrm{W}, 54^{\circ} 32^{\prime}$ 'S, 13. I. 2002.

18. PROVINCIA DE TIERRA DEL FUEGO, N. E. Ushuaia, Las Cotorras, $340 \mathrm{~m}, 68^{\circ} 00^{\prime} \mathrm{W}, 54^{\circ} 44^{\prime} \mathrm{S}, 13$. I. 2002.

19. PROVINCIA DE TIERRA DEL FUEGO, N. E. Ushuaia, Río Olivia, $400 \mathrm{~m}, 68^{\circ} 15^{\prime} \mathrm{W}, 54^{\circ} 46^{\prime} \mathrm{S}$, 14. I. 2002.

20. PROVINCIA DE TIERRA DEL FUEGO, E. Ushuaia, Puerto Almanza, 10 m, 670 $40^{\prime} \mathrm{W}, 54^{\circ} 51^{\prime}$ 'S, 15. I. 2002.

CHILE

21. XII REGIÓN, MAGALLANES, N. Villa Tehuelches, Puerta del Monte, $120 \mathrm{~m}, 71^{\circ} 20^{\prime} \mathrm{W}, 52^{\circ} 14^{\prime} \mathrm{S}, 17$. I. 2002.

22. XII REGIÓN, ÚLTIMA ESPERANZA, S. Puerto Natales, Las Lagunillas, $230 \mathrm{~m}, 7^{\circ} 05^{\prime} \mathrm{W}, 5^{\circ}{ }^{\circ} 53^{\prime} \mathrm{S}, 17$. I. 2002.

23. XII REGIÓN, ÚLTIMA ESPERANZA, N. Puerto Natales, Dos Lagunas, $180 \mathrm{~m}, 72^{\circ} 28^{\prime} \mathrm{W}, 51^{\circ} 22^{\prime}$ S, 17. I. 2002.

24. XII REGIÓN, ÚLTIMA ESPERANZA, Parque Nacional Torres del Paine, Refugio Lago del Toro, $100 \mathrm{~m}, 72^{\circ} 58^{\prime} \mathrm{W}$, $51^{\circ} 10^{\prime}$ 'S, 18. I. 2002

25. XII REGIÓN, ÚLTIMA ESPERANZA, Parque Nacional Torres del Paine, Pehoe, $120 \mathrm{~m}, 73^{\circ} 00^{\prime} \mathrm{W}, 51^{\circ} 05^{\prime} \mathrm{S}, 18$. I. 2002. 
26. XII REGIÓN, ÚLTIMA ESPERANZA, Parque Nacional Torres del Paine, Laguna Guanaco, $200 \mathrm{~m}, 72^{\circ} 50^{\prime} \mathrm{W}$, $51^{\circ} 01^{\prime}$ 'S, 18. I. 2002.

27. XII REGIÓN, ÚLTIMA ESPERANZA, Monumento Natural Cueva del Milodón, 200 m, $72^{\circ} 35^{\prime} \mathrm{W}, 5^{\circ} 33^{\prime} \mathrm{S}, 19$. I. 2002.

28. X REGIÓN, LLANQUIHUE, Ensenada, 80 m, 72³3'W, $41^{\circ} 14^{\prime}$ 'S, 25. I. 2002.

29. X REGIÓN, CHILOÉ, Chacao, $40 \mathrm{~m}, 73^{\circ} 32^{\prime} \mathrm{W}, 41^{\circ} 51^{\prime} \mathrm{S}$, 26. I. 2002.

30. X REGIÓN, CHILOÉ, Degan, Puente Puntra, $70 \mathrm{~m}$ $73^{\circ} 44^{\prime} \mathrm{W}, 42^{\circ} 13^{\prime}$ S, 26. I. 2002.

31. X REGIÓN, CHILOÉ, Isla Lemuy, Puqueldón, $50 \mathrm{~m}$, $73^{\circ} 40^{\prime} \mathrm{W}, 42^{\circ} 35^{\prime}$ 'S, 27. I. 2002.

32. X REGIÓN, CHILOÉ, Isla Lemuy, San Agustín, $80 \mathrm{~m}$,

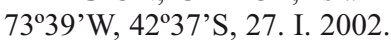

33. X REGIÓN, CHILOÉ, Hullinco, 150 m, 735ํ' $42^{\circ} 42$ 'S, 27. I. 2002.

34. X REGIÓN, CHILOÉ, Dalcahué, $90 \mathrm{~m}$, 73²40'W, $42^{\circ} 22^{\prime}$ 'S, 28. I. 2002.

35. X REGIÓN, CHILOÉ, Isla Quinchao, S. E. Achao, Putique, $90 \mathrm{~m}, 73^{\circ} 25^{\prime} \mathrm{W}, 42^{\circ} 32^{\prime} \mathrm{S}, 28$. I. 2002.

36. X REGIÓn, CHILOÉ, Isla Quinchao, N. W. Curaco de Vélez, Huya, 80 m, $73^{\circ} 37^{\prime} \mathrm{W}, 42^{\circ} 23^{\prime}$ S, 28. I. 2002.

37. X REGIÓN, CHILOÉ, Mocopulli, $70 \mathrm{~m}, 73^{\circ} 43^{\prime} \mathrm{W}$, $42^{\circ} 22^{\prime}$ 'S, 28. I. 2002.

38. X REGIÓN, LLANQUIHUE, E. Lenca, Chaica, $500 \mathrm{~m}$, $72^{\circ} 37^{\prime} \mathrm{W}, 41^{\circ} 36^{\prime} \mathrm{S}, 29$. I. 2002.

39. X REgión, LlanQUiHue, N. E. Puerto Montt, Correntoso, $220 \mathrm{~m}, 72^{\circ} 45^{\prime} \mathrm{W}, 4^{\circ} 21^{\prime}$ 'S, 29. I. 2002.

40. X REGIÓN, LLANQUIHUE, Frutillar Bajo, $60 \mathrm{~m}$, $73^{\circ} 02^{\prime} \mathrm{W}, 41^{\circ} 07^{\prime} \mathrm{S}, 30$. I. 2002.

41. X REGIÓN, OSORNO, N. Puerto Octay, Carril, $100 \mathrm{~m}$, $72^{\circ} 51^{\prime} \mathrm{W}, 40^{\circ} 56^{\prime} \mathrm{S}, 30$. I. 2002.

42. X REGIÓN, OSORNO, E. Puerto Octay, La Picada, Puente del Río Blanco, $250 \mathrm{~m}, 72^{\circ} 33^{\prime} \mathrm{W}, 41^{\circ} 00^{\prime} \mathrm{S}, 30$. I. 2002.

43. X REGIÓN, OSORNO, E. Entre Lagos, Futachín, $280 \mathrm{~m}$, $72^{\circ} 24^{\prime} \mathrm{W}, 40^{\circ} 43^{\prime} \mathrm{S}, 31$. I. 2002

ARGENTINA

44. PROVINCIA DE RIO NEGRO, N. El Bolsón, El Foyel, $800 \mathrm{~m}, 71^{\circ} 26^{\prime} \mathrm{W}, 41^{\circ} 48^{\prime} \mathrm{S}, 4$. II. 2002.

45. PROVINCIA DE RIO NEGRO, N. El Bolsón, Los Repollos, Cerro Saturnino, $655 \mathrm{~m}, 71^{\circ} 25^{\prime} \mathrm{W}, 41^{\circ} 50^{\prime} \mathrm{S}, 4$ II. 2002.

46. PROVINCIA DE CHUBUT, S. E. El Bolsón, El Hoyo, $330 \mathrm{~m}, 71^{\circ} 27^{\prime} \mathrm{W}, 42^{\circ} 05^{\prime} \mathrm{S}$, 5. II. 2002.

47. PROVINCIA DE CHUBUT, S. W. El Bolsón, Lago Puelo, $510 \mathrm{~m}, 71^{\circ} 40^{\prime} \mathrm{W}, 42^{\circ} 10^{\prime} \mathrm{S}, 5$. II. 2002

48. PROVINCIA DE CHUBUT, Lago Epuyén, Puerto Patriada, $640 \mathrm{~m}, 71^{\circ} 30^{\prime} \mathrm{W}, 42^{\circ} 13^{\prime} \mathrm{S}$, 6. II. 2002.

49. PROVINCIA DE RIO NEGRO, N. El Bolsón, El Foyel, $800 \mathrm{~m}, 71^{\circ} 26^{\prime} \mathrm{W}, 41^{\circ} 48^{\prime} \mathrm{S}, 7$. II. 2002.

50. PROVINCIA DE NEUQUÉN, Villa La Angostura, $760 \mathrm{~m}$, $71^{\circ} 40^{\prime} \mathrm{W}, 40^{\circ} 47^{\prime} \mathrm{S}, 8$. II. 2002.

51. PROVINCIA DE RIO NEGRO, Paso Chacabuco, Río Limay, $510 \mathrm{~m}, 71^{\circ} 02^{\prime} \mathrm{W}, 40^{\circ} 45^{\prime} \mathrm{S}, 9$. II. 2002.

52. PROVINCIA DE BUENOS AIRES, Médanos, Villarino, $25 \mathrm{~m}, 62^{\circ} 54^{\prime} \mathrm{W}, 38^{\circ} 46^{\prime} \mathrm{S}, 28$. II. 2002.

53. PROVINCIA DE BUENOS AIRES, Bahía Blanca Ciudad, $20 \mathrm{~m}, 62^{\circ} 11^{\prime} \mathrm{W}, 38^{\circ} 44^{\prime} \mathrm{S}$. Varias fechas según se anota en cada caso.
El material era capturado mangueando la vegetación arbórea y arbustiva existente en cada localidad, y ocasionalmente se ha recolectado algún material atraído a la luz durante la noche. Para cada especie se citan las localidades y fechas de captura según la numeración anteriormente listada, el número de Or O’, $\bigcirc$ ○ o larvas capturados y el sustrato vegetal sobre el que han sido hallados los ejemplares. Algunos adultos fueron mantenidos en cajas de cultivo de $4 \times 4 \times 1^{\prime} 5 \mathrm{~cm}$ a temperatura ambiente para la obtención de puestas y se les proporcionó humedad mediante un algodón humedecido en agua y áfidos para su alimentación. Para la descripción de la morfología y quetotaxia larvaria éstas se aclaraban previamente en ácido láctico.

El material que se cita queda depositado en la colección del autor ubicada en el Departamento de Zoología y Antropología Física de la Universidad Complutense de Madrid (España ) salvo algunos ejemplares que se han depositado en el Museo Nacional de Ciencias Naturales de Madrid (MNCNM), Museo Argentino de Ciencias Naturales "Bernardino Rivadavia" de Buenos Aires (MACN) y Museo Nacional de Historia Natural de Santiago de Chile (MNHNS).

Para la sistemática se sigue la ordenación general dada por Oswald (1993 a), para la terminología general se sigue la empleada usualmente en esta familia (Tjeder, 1961 y Aspöck et al., 1980) y para la identificación de las especies de árboles y plantas citadas se ha utilizado Hoffmann (1997), Erize (2000 a, b) y Haene \& Gostaro (2001).

\section{Material estudiado}

Hemerobiinae Latreille, 1802

\section{Hemerobius bolivari Banks, 1910}

Especie ampliamente distribuida por la Región Neotropical, de morfología y pigmentación relativamente variable y muy eurioica en la selección de su sustrato vegetal y altitudinal según indica Monserrat (1996) y corroboran los datos obtenidos.

Material ESTUDiAdO: 1: 10 ○ ○", 2 ᄋ +2 larvas. 2:

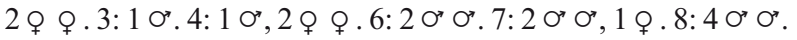

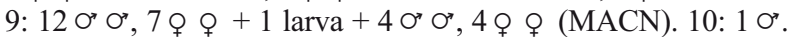

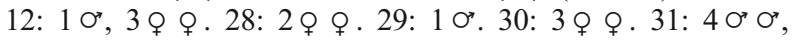
2 우 ㅇ. . 32: 1 ᄋ", 2 우 ㅇ. . 35: 1 ᄋ", 3 우 ㅇ. . 36: 1 ㅇ. . 39: 1 ㅇ. . 40:

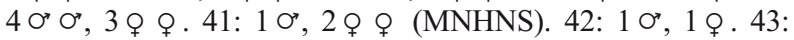

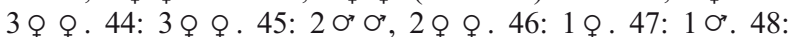

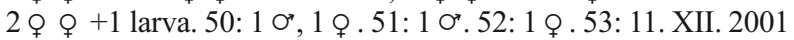

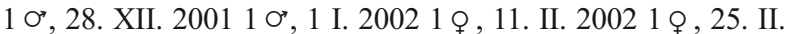
20021 \%, 25. II. 20021 ơ ex ovo, 28. IV. 20021 ơ ex ovo. 
Se han recolectado ejemplares sobre una gran variedad de sustratos vegetales, hecho que la diferencia de $H$. chilensis, especie mucho más estenoica y circunscrita a Nothofagus. De ellos se han capturado 49 imagos + 2 larvas sobre Pinus halepensis Mill., 12 imagos sobre Luma apiculata (Dc.), 7 sobre Pilgerodendron oviferum (Don.) Flor, 7 sobre Nothofagus pumilio (Poepp. et Endel.), 7 a la luz, 5 sobre Austrocedrus chilensis (Don.) Florin et Boutleje, 4 sobre Nothofagus dombeyi (Mirbel), 4 sobre Nothofagus nitida (Phil.), 3 sobre Tepualia stipularis (Hook, Fil) Griseboch, 3 sobre Drimys winteri Forst, 3 sobre Crataegus monogyna Jacq, 3 imagos +1 larva sobre Pinus radiata D. Don, 3 imagos sobre Prosopis caldenia Bork., 2 sobre Chusquea culeou E. Desk, 2 sobre Desfontainia spinosa R. et P., 2 sobre Jordina rhombifolia Hook et Arn., 2 sobre Tamarix gallica L., 2 y una larva sobre Pinus radiata D. Don., 1 sobre Schimus fasciculatus (Gr.) Johnst, 1 sobre Amomirthus luma (Mol.) Legrand y 1 errante.

Algunos adultos capturados en la localidad 53 que fueron mantenidos en cajas de cultivo permanecieron vivos entre 14 y 22 días, observándose una marcada actividad en la búsqueda de alimento, especialmente en las horas del crepúsculo y durante la noche. En base a huevos puestos por algunos de estos ejemplares se ha completado el ciclo de su desarrollo larvario en condiciones de laboratorio y junto a las larvas capturadas podemos pasar a la descripción de sus fases juveniles que eran desconocidas.

Una o capturada a la luz el 25. II. 02 realizó varias puestas a lo largo de 14 días, una durante la primera noche de 19 huevos, otra cinco días después de 36 huevos y 5 huevos más puestos aisladamente durante los restantes días.

Los huevos eran depositados aisladamente, no en posición vertical perpendicular al sustrato sino adheridos longitudinalmente sobre la tapa de la caja de cultivo o el algodón que alojaba. Eran de color pardo pálido y tornan a pardo más oscuro conforme el desarrollo embrionario progresa. El micropilo es esférico, poco prominente y la superficie del corion porta impresiones foliculares poco aparentes. Completado su desarrollo embrionario practican una pequeña fisura longitudinal desde el micropilo. Eclosionan a los 4-5 días y se les proporcionó para su alimentación ninfas e imagos de Brachycaudus (Appelia) schwartzi (Börner, 1931) y Aphis (Aphis) gossypii gossypii Glover, 1877.

Las larvas neonatas permanecen sobre el corion un tiempo antes de iniciar su actividad. Se desplazan arqueando y haciendo palanca sobre el sustrato con el extremo del abdomen, mueven lateralmente a uno y otro lado su cabeza con el fin de localizar presas y mientras comen arquean las antenas hacia atrás. Tras la ingesta dedican unos minutos al aseo, principalmente de las antenas. Son muy activas y poco huidizas y tienden a permanecer en momentos de reposo sobre o bajo las hojas. Realizan la primera muda a los 4 días y según los datos anotados completan su desarrollo larvario entre 32-35 días desde la eclosión del huevo, tras los cuales inician la fabricación del capullo y 9-11 días después emergen como imagos.

Los neonatos (Fig. 1) tienen tegumento pardo muy pálido, su cápsula cefálica más oscura, especialmente sobre una banda lateral y aparentemente poseen dos pequeños estemata anteriores y uno mayor posterior, todos de color negro. Piezas bucales pálidas, algo más oscuras las antenas. Tergo torácico pálido con dos bandas oscuras, una a cada lado de una amplia banda media pálida y una leve banda transversal pálida sobre las antecostas. Patas pardo pálidas, con el tercio basal de los fémures y los dos tercios distales de las tibias más pálidos. Abdomen pálido con dos líneas laterales longitudinales formadas por bandas trasversales irregulares más oscuras en la zona dorso- lateral de los segmentos, éstas dejan una zona circular pálida alrededor de los estigmas bajo la cual se dispone otra hilera de pequeñas manchas pardas. Quetotaxia, pigmentación estructural tegumentaria y morfología general según figuras 1-3.

Las larvas de segundo y tercer estadio (Figs. 4, $5,32,33)$ son pardas más oscuras. Cápsula cefálica con márgenes laterales pardos muy oscuros y una banda fusiforme parda sobre la línea media, así como dos triangulares sobre el vértex. Aparentemente dos pequeños estemata anteriores $\mathrm{y}$ uno mayor posterior, todos de color negro. Escapo pardo, algo más oscuro que el flagelo. Mandíbulas, maxilas y palpos pardo pálidos, último palpómero labial más oscuro, piriforme y porta una porción membranosa ventral elíptica apical.

Tórax y abdomen con una banda longitudinal amarillenta lechosa y dos líneas pardas longitudinales, una a cada lado, más intensas en el tórax y más irregulares y difusas en los segmentos del abdomen. Escleritos del tergo protorácico arqueados, los del meso- y metatórax claviformes, mucho más pequeños. Patas pardo oscuras con los trocánteres, el cuarto basal de los fémures y los tres cuartos distales de las tibias más pálidos. Escleritos distales del abdomen pardo oscuros. Quetotaxia, pigmentación estructural tegumentaria y morfología general según figuras 4-7. 


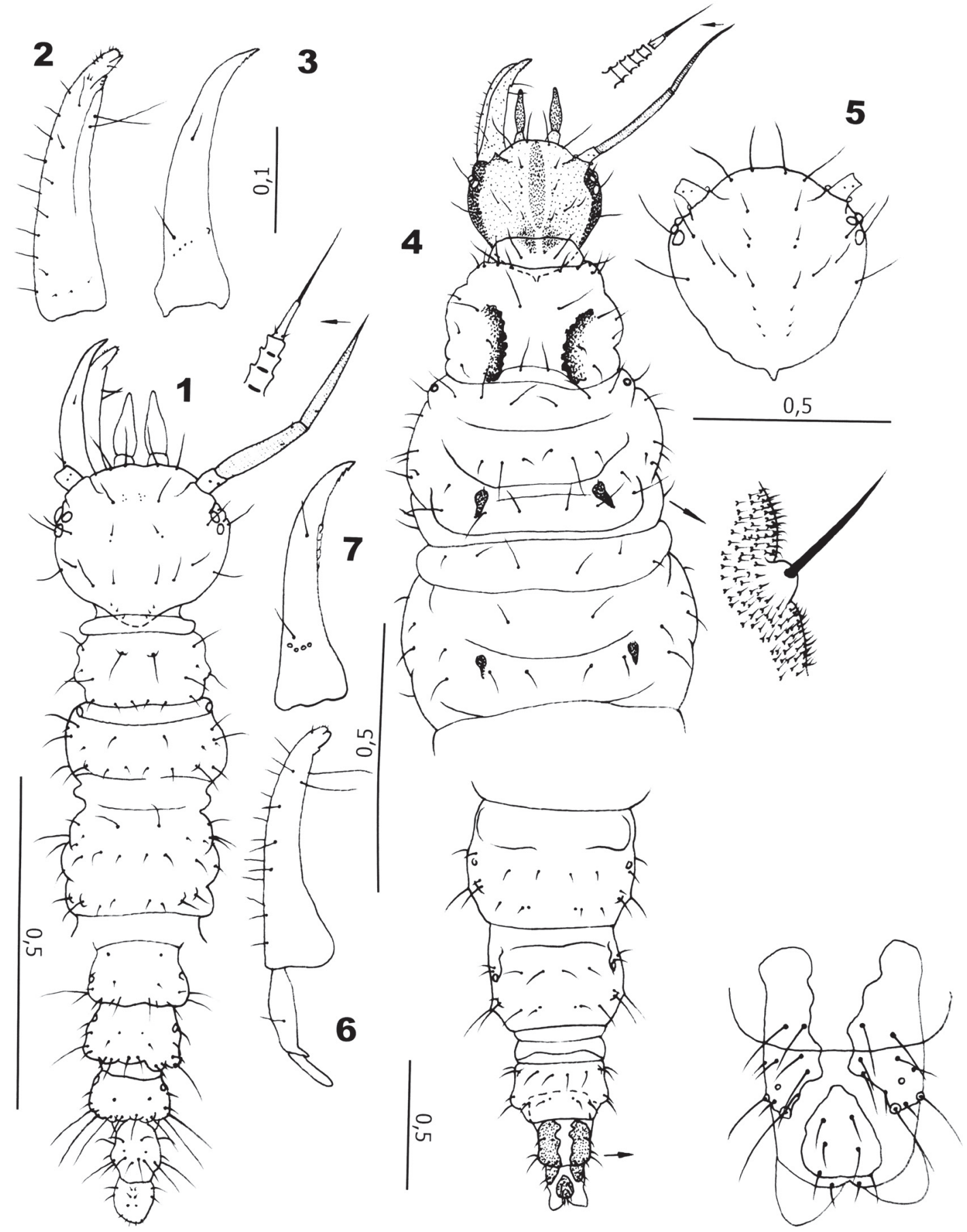

Figs. 1-7.- Hemerobius bolivari. 1: Quetotaxia dorsal de la cabeza, tórax y extremo abdominal de la larva neonata, ampliado extremo antenal. 2: Maxila, dorsal. 3: Mandíbula, dorsal. 4: Quetotaxia dorsal de la cabeza, tórax y extremo abdominal de la larva desarrollada, ampliados extremo antenal, abdominal y una pequeña porción del tegumento. 5: Cápsula cefálica, dorsal. 6: Maxila, dorsal. 7: Mandíbula, dorsal. Escala en mm.

Figs. 1-7.- Hemerobius bolivari. 1: Dorsal chaetotaxy of head, thorax and abdominal tip of the neonate larva, magnified antenal tip. 2: Maxilla, dorsal. 3: Mandible, dorsal. 4: Dorsal chaetotaxy of head, thorax and abdominal tip of the full grown larva, magnified antenal and abdominal tip and a little portion of tegument. 5: Cephalic capsule, dorsal. 6: Maxilla, dorsal. 7: Mandible, dorsal. Scale in $\mathrm{mm}$. 


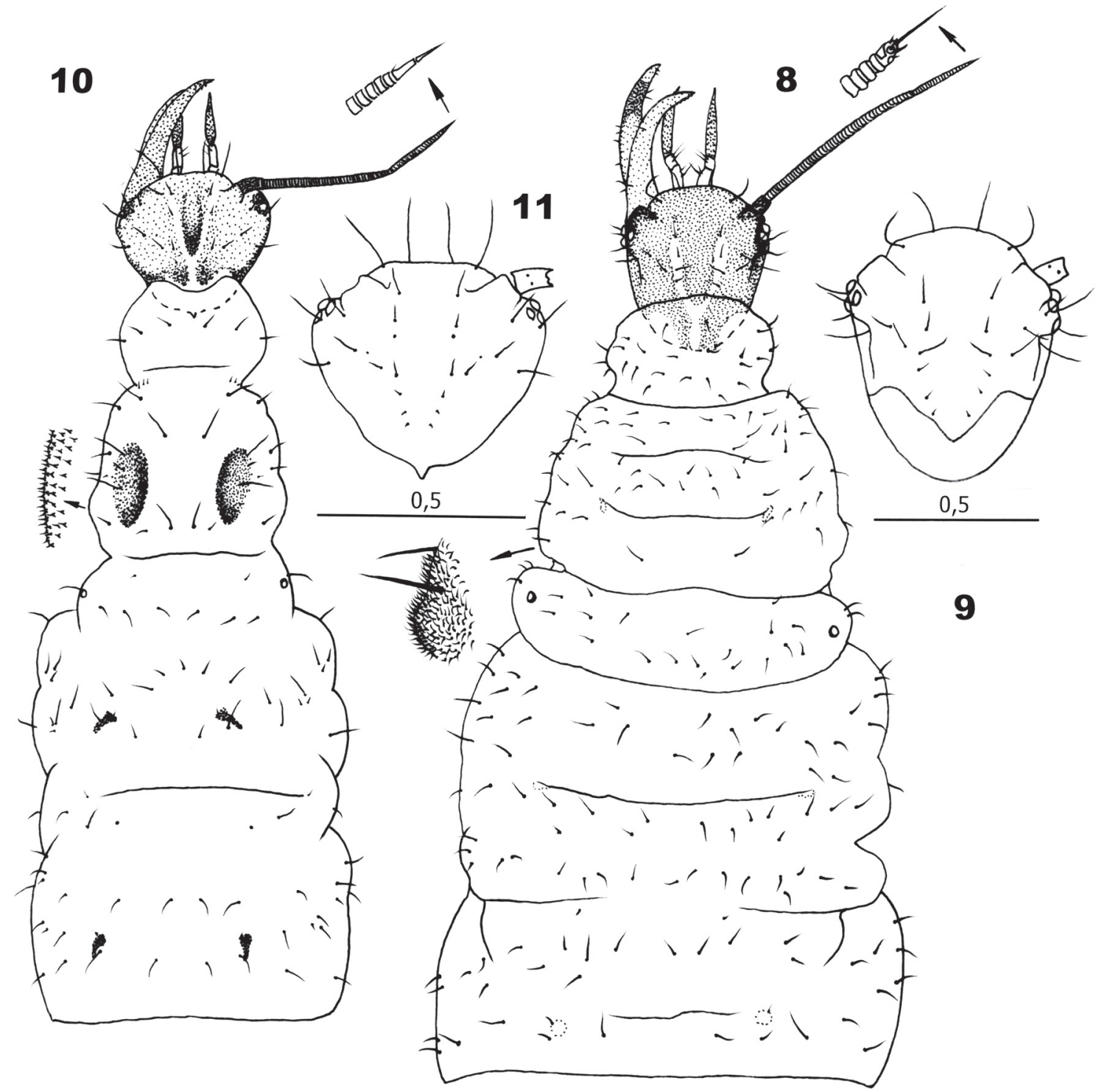

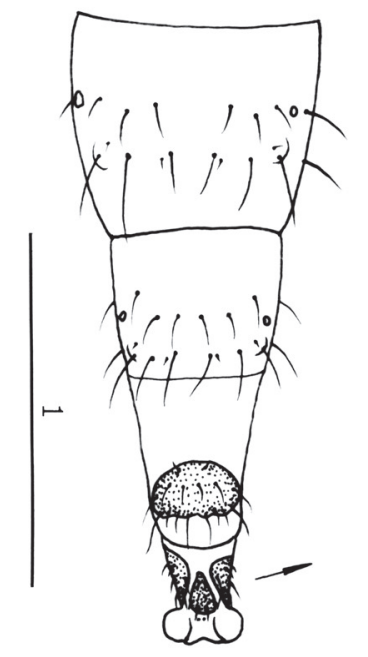
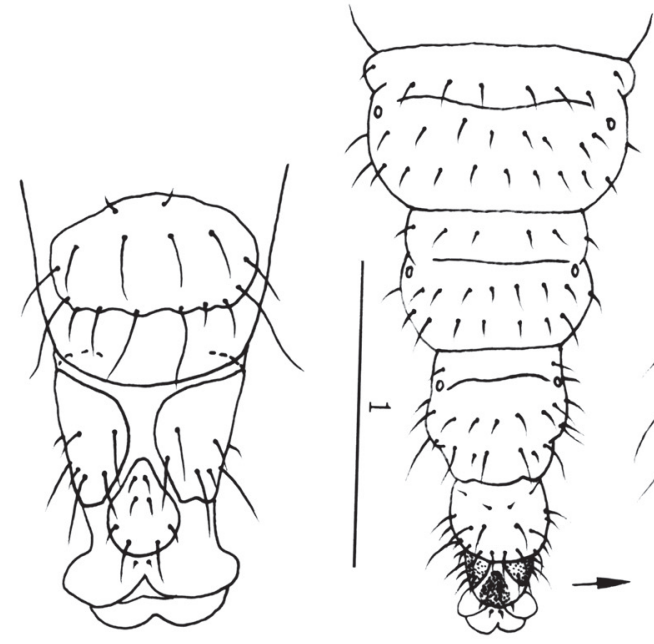

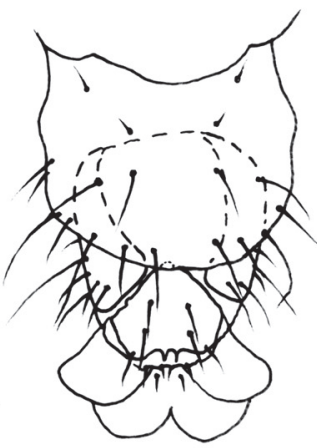




\section{Hemerobius chilensis Nakahara, 1965}

Especie andina conocida de Chile, Argentina y Perú, vinculada a los bosques de Nothofagus.

MATERIAL ESTUDIADO: 13: 4 ㅇ․ $14: 10^{\prime}+2$ larvas. 15: 17 on ○’, 11 ᄋ o +1 larva. 16: 2 ơ on, 3 ᄋ o + 1 larva. 17:

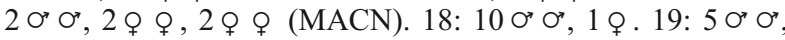

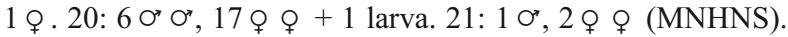
22: 13 ○’ ○’, 13 ○ +1 larva. 23: 29 ơ ơ 19 \% + 1 larva,

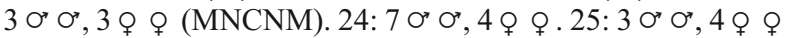

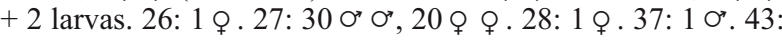
3 우. ․ 49: $10^{\rtimes}, 2$ 우 ㅇ․

Se han recolectado 141 imagos y 7 larvas sobre Nothofagus pumilio (Poepp. et Endel.), 55 imagos y 2 larvas sobre Nothofagus antarctica (Forst.), 18 imagos sobre Nothofagus betuloides (Mirbel) Blume, 2 sobre Nothofagus dombeyi (Mirbel) y 2 sobre Chusquea culeou E. Desk que abrumadoramente corroboran su asociación con Nothofagus anteriormente citada.

Los estadios preimaginales de esta especie eran desconocidos. En base las larvas anteriormente citadas, que se recolectaron junto a imagos de esta especie, se describen sus estadios juveniles y se comparan con los de Hemerobius bolivari que se han descrito anteriormente. Las diferencias halladas en los estadios larvarios entre estas dos especies contribuyen a la defensa de Hemerobius chilensis como una especie distinta y válida.

Las larvas de segundo $\mathrm{L}_{2} \mathrm{y}$ de tercer estadio $\mathrm{L}_{3}$ (Figs. 8, 9, 34, 35) son pardas, más oscuras dorsalmente. La cápsula cefálica es parda con márgenes laterales más oscuros y una zona en Y sobre la sutura media más pálida. Aparentemente dos pequeños estemata posteriores y uno algo mayor anterior, todos de color negro. Escapo pardo, algo más oscuro que el flagelo. Mandíbulas pardas, maxilas notablemente más oscuras en su tercio distal salvo el ápice que es pálido y palpos labiales pardo pálidos con último palpómero más oscuro.

Pronoto irregularmente pardo, con una estrecha banda longitudinal pálida y dos líneas pálidas en zigzag oblicuas, una a cada lado. Margen posterior del pronoto y anterior del mesonoto formando una llamativa mancha rectangular pálida. Meso- y metanoto irregularmente pardo con una mancha pálida tras el estigma, circular en $\mathrm{L}_{2} \mathrm{e}$ irregularmente circular en $\mathrm{L}_{3}$, margen posterior del mesonoto $\mathrm{y}$ anterior del metanoto formando una llamativa mancha rectangular pálida. Escleritos de los tergos torácicos apenas desarrollados. Patas uniformemente pardo oscuras, levemente más oscura la mitad distal de los fémures. Tergos abdominales con manchas pardas irregularmente fragmentadas, dejando una irregular línea media pálida, más extensa conforme los segmentos son más caudales, lateralmente una hilera de manchas pálidas, circulares en $\mathrm{L}_{2} \mathrm{e}$ irregulares en $\mathrm{L}_{3}$, acompañan a los estigmas. Escleritos distales del abdomen pardo oscuros. Pro- y mesoesterno pardo muy pálido, metaesterno y esternos abdominales pardos. Quetotaxia, pigmentación estructural tegumentaria y morfología general según figuras 8 y 9 .

Discusión: Son numerosas las descripciones de larvas pertenecientes al género Hemerobius que en su mayoría pertenecen a especies Paleárticas, Neárticas u Holárticas, sin embargo, no se conocen los estadios larvarios en ninguna de las 30 especies neotropicales de este género (Monserrat, 1996; Oswald \& Tauber, 2001).

Como era de esperar y debido a la similitud morfológica de sus imagos, el aspecto y quetotaxia de los estadios juveniles de esta especie es muy similar al de Hemerobius bolivari, sin embargo su pigmentación cefálica y especialmente el resto de la tegumentaria es muy diferente en ambas especies. En Hemerobius bolivari la línea dorsal pálida en el tergo torácico y abdominal es continua, ancha, bien delimitada y uniforme, mientras que en $\mathrm{H}$. chilensis es tenue, difusa, irregular y extendida entre pro- y mesonoto y entre meso- y metanoto en dos manchas rectangulares pálidas muy llamativas (Figs. 32-35). Además en $H$. chilensis la cabeza es mucho más alargada, los escleritos tergales del tórax apenas son visibles, las tibias son uniformemente pardas y la quetotaxia (tanto micro- como macrotriquias) es mucho más abundante en general

Figs. 8-11.- Hemerobius chilensis. 8: Quetotaxia dorsal de la cabeza, tórax y extremo abdominal de la larva desarrollada, ampliados extremo antenal, abdominal y una pequeña porción del tegumento. 9: Cápsula cefálica, dorsal. Hemerobius stenopterus. 10: Quetotaxia dorsal de la cabeza, tórax y extremo abdominal de la larva desarrollada, ampliados extremo antenal, abdominal y una pequeña porción del tegumento. 11: Cápsula cefálica, dorsal. Escala en mm.

Figs. 8-11.- Hemerobius chilensis. 8: Dorsal chaetotaxy of head, thorax and abdominal tip of the full grown larva, magnified antenal and abdominal tip and a little portion of tegument. 9: Cephalic capsule, dorsal. Hemerobius stenopterus. 10: Dorsal chaetotaxy of head, thorax and abdominal tip of the full grown larva, magnified antenal and abdominal tip and a little portion of tegument. 11: Cephalic capsule, dorsal. Scale in $\mathrm{mm}$. 
y las setas son de menor longitud y se disponen en tres, y no dos, anillos en los segmentos abdominales (Figs. 4, 8).

\section{Hemerobius stenopterus Monserrat, 1996}

Especie conocida de la zona andina meridional de Chile y Argentina. Su biología es prácticamente desconocida.

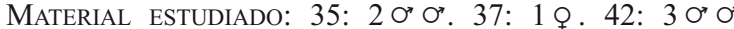

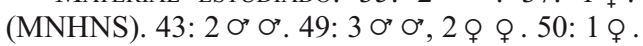

Parece habitar en sotobosques valdivianos muy húmedos, sombríos y cerrados. Se han capturado 3 ejemplares sobre Desfontainia spinosa R. et P., 2 sobre Nothofagus pumilio (Poepp. et Endel.), 2 sobre Nothofagus nitida (Phil.) y 1 sobre Nothofagus dombeyi (Mirbel), aunque también se han recolectado 5 ejemplares en zonas más abiertas al borde de un lago sobre colihue bambú: Chusquea culeou E. Desk y 1 ejemplar a la luz. Los datos anotados parecen corroborar la escasa orofilia citada para esta especie por Monserrat (1996) habiéndose ahora recolectado ejemplares entre los 70-800 m.

Una larva de comportamiento y movimientos muy ágiles, capturada en la localidad 32 junto con ejemplares adultos de Hemerobius bolivari en un medio muy húmedo de sotobosque de Luma apiculata (Dc.) muy similar al de otras localidades donde se han capturado imagos de Hemerobius stenopterus, pertenece sin duda al género Hemerobius, pero por su mayor agilidad y su aspecto general no parece pertenecer ni a $H$. bolivari ni a $H$. chilensis que acaban de ser descritas, y debido a su apariencia mucho más estilizada, a la mayor longitud de sus escapos, a su cabeza más triangular y ancha, al mayor desarrollo de los escleritos del tergo protorácico y a su quetotaxia (Figs. 10,11) no se corresponde con ninguna de las dos especies citadas y, por exclusión, deducimos que muy probablemente pertenezca a la especie que tratamos.

\section{Sympherobiinae Comstock, 1918}

Nomerobius psychodoides (Blanchard en Gay, 1851)

Especie citada de Chile, Argentina, Perú, Uruguay y Brasil, y de biología casi desconocida.

Material estudiado: 2: 2 우. 5: $20^{\circ} 0^{\circ}, 1$ ㅇ. . 6: 2 우. 7: 1 ㅇ. . 11: 1 ㅇ. 12:3우 ㅇ. 46: 2 o" on.

Oswald (1990) y Monserrat (1998) recogen las escasas citas conocidas sobre la biología de esta especie que parece estar asociada a zonas secas y ha sido citada sobre Prosopis y Larrea. Nosotros hemos recolectado 6 ejemplares sobre Prosopis cal- denia Bork., 4 sobre Pinus halepensis Mill., 3 a la luz y 1 sobre Acacia caven (Molina). En la línea de lo anotado por Monserrat (1998) las membranas peritróficas de algunos de estos ejemplares portaban restos de pequeños artrópodos, principalmente pulgones y aleiródidos, escamas de mariposas, hifas de hongos y polen diverso incluido de coníferas.

\section{Nomerobius signatus (Hagen, 1861)}

Especie conocida de Chile, Argentina y Perú, de biología casi desconocida ya que la mayor parte de los ejemplares anteriormente conocidos se habían recolectado a la luz o en trampas de Malaise, generalmente en zonas xéricas.

Material estudiado: 45: $40^{\circ} 0^{\circ}, 6$ \% $~ . .51: 10^{\circ}, 1$ q.

Todos recolectados sobre Austrocedrus chilensis (Don.) Florin et Boutleje, lo que sugiere su asociación con esta cupresácea y en la línea de lo anotado por Monserrat (1998) las membranas peritróficas de algunos de estos ejemplares portaban abundantes restos de áfidos y en menor cantidad hifas de hongos, esporas y polen diverso.

\section{Nomerobius cuspidatus Oswald, 1990}

Especie conocida de Argentina, Bolivia y Chile. Su biología es desconocida.

Material eSTUDIADO: 6: 1 larva. 46: 1 o . 53: 12. XII. 2001

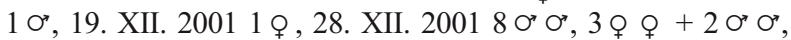
2 의 (MACN), 1. I. 20021 ㅇ, 2. I. 20021 ㅇ, 11. II. 2002

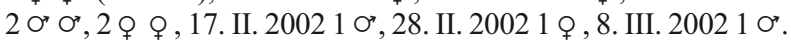

Todos los ejemplares en estado de imago han sido capturados a la luz, por lo que poco podemos aportar a su casi desconocida biología. La larva citada y asignada a esta especie (Fig. 36) se capturó sobre Tamarix gallica. En la línea de lo anotado por Monserrat (1998) las membranas peritróficas de alguno de los imagos estudiados portaban abundante material silíceo, también restos de pequeños artrópodos, hifas de hongos, esporas y polen diverso.

Se han observado ejemplares adultos comiendo áfidos y ayudándose activamente con el primer par de patas que utilizan a modo de patas raptoras para sujetar a su presa, las fuertes setas presentes en la cara interna del fémur y las laxas articulaciones de los proporcionalmente largos tarsómeros posibilitan morfológica y funcionalmente este comportamiento. Tras la ingesta de un pulgón dedican unos minutos al aseo, especialmente de las antenas, pero también de las piezas bucales y patas anteriores antes de atacar un nuevo pulgón, tras cuya ingesta vuelven a practicar el citado aseo y repiten este comportamiento una y otra vez tras cada ingesta de 
una presa. Parece deducirse la existencia de glándulas en la zona lateral del abdomen, a tenor del frecuente roce del tercer par de patas con esta zona durante el aseo. Algunos adultos que fueron mantenidos en cajas de cultivo permanecieron vivos hasta 20 días, observándose una marcada actividad en la búsqueda de alimento, especialmente en las horas del crepúsculo y durante la noche.

Los estadios preimaginales de este género / especie son desconocidos y a partir de algunos ejemplares recolectados en la localidad 53 se obtuvieron varias puestas que han permitido completar su desarrollo larvario y en base a estos ejemplares y a la larva capturada podemos describir sus fases juveniles.

Un ejemplar o capturado el 1.I.02 realizó una puesta de 3 huevos esa noche y otra $\$$ capturada el 28.II.02 realizó una puesta de 6 huevos el 1.III.02 y otra de 3 huevos el 5.III.02. Los huevos eran depositados aisladamente, no en posición vertical perpendicular al sustrato, sino adheridos longitudinalmente, junto a las nerviaciones del envés de las hojas donde se alojaban las colonias de áfidos proporcionadas como alimento al imago. Eran de color blancuzco y tornan a pardo conforme el desarrollo embrionario progresa. El micropilo es esférico, poco prominente y la superficie del corion porta pequeñas impresiones foliculares. Completado su desarrollo embrionario practican una pequeña fisura longitudinal desde el micropilo. Eclosionan a los 7 días y se les proporcionó para su alimentación ninfas e imagos de Brachycaudus (Appelia) schwartzi (Börner, 1931) y Aphis (Aphis) gossypii gossypii Glover, 1877.

Las larvas neonatas permanecen sobre el corion un tiempo antes de iniciar su actividad. Se desplazan arqueando y haciendo palanca con el extremo del abdomen, mueven lateralmente a uno y otro lado su cabeza con el fin de localizar presas, pueden atacar a presas (áfidos) mucho mayores que ellas y mientras comen arquean las antenas hacia atrás. Tras la ingesta dedican unos minutos al aseo, principalmente de las antenas. Son muy activas e huidizas y tienden a esconderse en fisuras y repliegues de las hojas o bajo ellas o bajo cualquier objeto para no estar expuestas, por lo que son de muy difícil localización. Este comportamiento se extrema conforme las larvas se desarrollan y al final de su desarrollo son especialmente huidizas, tendiendo permanentemente a refugiarse y permanecer bajo objetos o en los repliegues de las hojas, hecho que sugiere un cierto comportamiento corticícola. Realizan la primera muda a los 4 días y según los datos anotados completan su desarrollo larvario en
12-14 días desde la eclosión del huevo, tras los cuales inician la fabricación del capullo.

Los neonatos (Figs. 12-14) tienen tegumento cefálico pardo pálido, más oscura una banda lateral en la cápsula cefálica. Una glándula dorsal de aspecto irregular, probablemente digestiva parece alojarse en la mitad posterior de la cabeza. Piezas bucales muy pálidas, algo más oscuras las antenas. Aparentemente poseen tres estemas anteriores, negros, pequeños y en arco y uno mayor en posición más retrasada. Patas muy pálidas. Tergo torácico pálido en su banda media dorsal y a ambos lados de ella existe una ancha estría trasversal parda oscura sobre la zona anterior de cada uno de los tres segmentos, especialmente oscuras en meso- y metanoto, éstas estrías se unen entre sí por una banda longitudinal algo más pálida que se arquea dejando una zona circular blancuzca en el margen lateral exterior del pronoto y mesonoto. Setas dorsales del tórax frecuentemente ensanchadas en embudo en su extremo y de reborde estrellado. Abdomen pálido con dos líneas laterales longitudinales formadas por bandas trasversales pardas más oscuras en la zona dorso- lateral de sus segmentos. Éstas están interrumpidas entre los segmentos II / III, dejando una banda oval pálida dorsal y son continuas en los segmentos posteriores. Quetotaxia, pigmentación estructural tegumentaria y morfología general según figuras 12-14.

Las larvas de segundo y tercer estadio (Figs. 15$18,36)$ son pardas mucho más oscuras, casi negras, alternadas con zonas llamativamente blancas que le dan una coloración evidentemente disruptiva. La cápsula cefálica está parcialmente embutida en el pronoto, es pálida dorsalmente con una tenue doble línea grisácea sobre la zona media y está flanqueada oblicuamente a ambos lados por una banda lateral, mucho más oscura, casi negra, que se continúa hacia el tórax. Aparentemente tres estemas anteriores e internos en arco y uno mayor más lateral. Antenas pardas, con escapo más oscuro, mandíbulas, maxilas y palpos pardo pálidos, más oscuro el penúltimo segmento del palpo. Tórax con una banda pálida grisácea sobre la línea media dorsal que se hace más difusa hacia meso- y metanoto. Lateralmente los tres segmentos torácicos son pardos muy oscuros, casi negros en el meso- y metanoto, los tubérculos laterales del pro- y mesonoto portan una llamativa mancha blanca circular que en el metanoto se alarga y adelgazan progresivamente conforme se dirigen hacia la línea media dorsal creando una llamativa banda blanca trasversal entre ambos tagmas. Patas pardas. Abdomen pardo grisáceo, más rojizo en los dos tercios posteriores. Los 

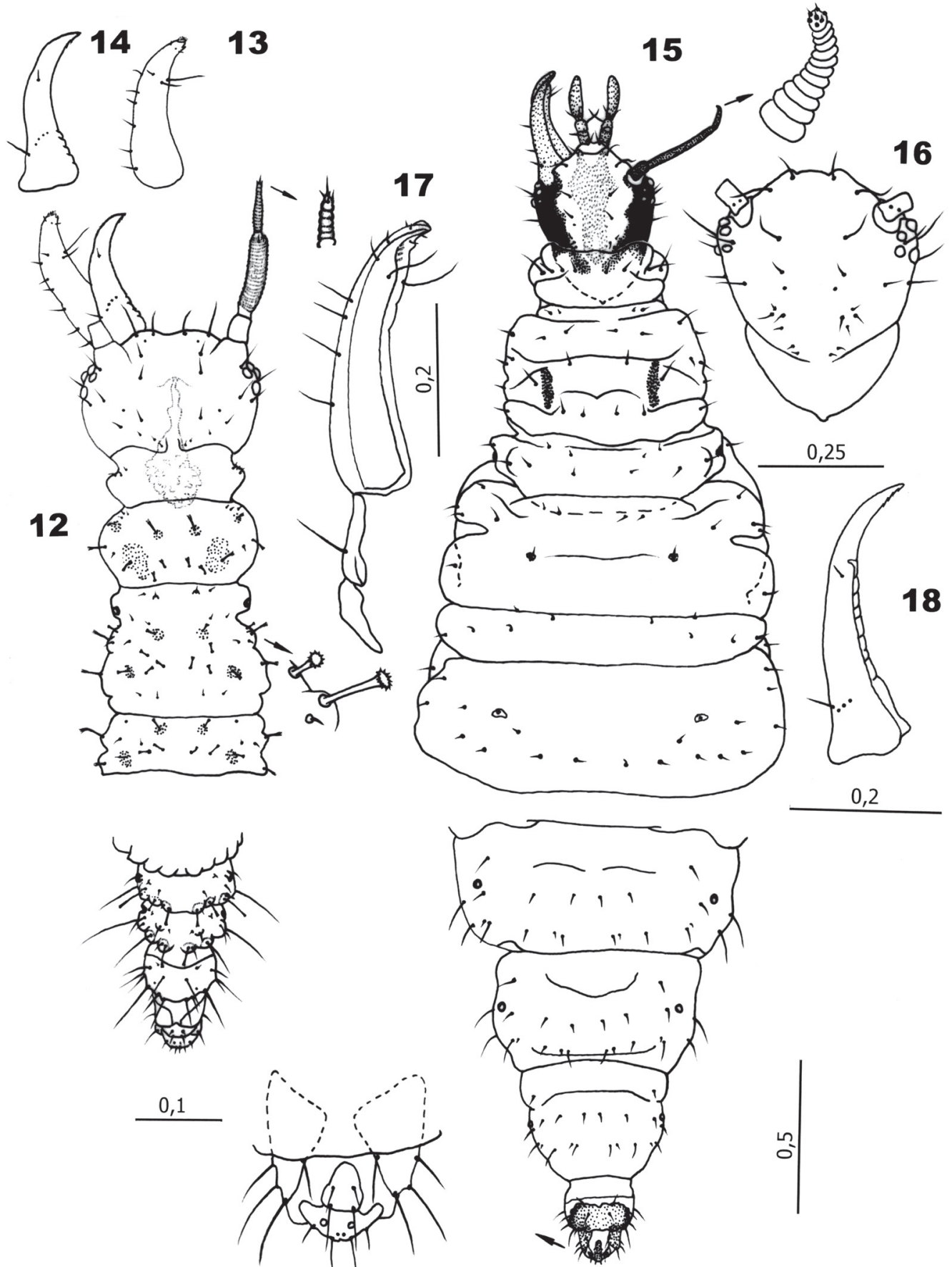

Figs. 12-18.- Nomerobius cuspidatus. 12: Quetotaxia dorsal de la cabeza, tórax y extremo abdominal de la larva neonata, ampliadas tres setas y extremo antenal. 13: Maxila, dorsal. 14: Mandíbula, dorsal. 15: Quetotaxia dorsal de la cabeza, tórax y extremo abdominal de la larva desarrollada, ampliados extremo antenal y abdominal. 16: Cápsula cefálica, dorsal. 17: Maxila, dorsal. 18: Mandíbula, dorsal. Escala en mm.

Figs. 12-18. - Nomerobius cuspidatus. 12: Dorsal chaetotaxy of head, thorax and abdominal tip of the neonate larva, magnified three setae and antenal tip. 13: Maxilla, dorsal. 14: Mandible, dorsal. 15: Dorsal chaetotaxy of head, thorax and abdominal tip of the full grown larva, magnified antenal and abdominal tip. 16: Cephalic capsule, dorsal. 17: Maxilla, dorsal. 18: Mandible, dorsal. Scale in $\mathrm{mm}$. 
segmentos de la mitad distal portan una llamativa mancha circular blanca en su región lateral y así como pequeñas manchas blancas a ambos lados de su línea media dorsal. Quetotaxia, pigmentación estructural tegumentaria y morfología general según figuras 15-18.

Discusión: Los estadios larvarios del género Nomerobius Navás, 1915 eran desconocidos (Oswald, 1993a, Oswald \& Tauber, 2001) y dentro de la subfamilia Sympherobiinae Comstock, 1918, a la cual pertenece, sólo existen datos del género Sympherobius Banks, 1904, en particular de especies mayoritariamente Paleárticas y Neárticas (Oswald, 1993 a, Oswald \& Tauber, 2001).

Los datos aquí aportados indican semejanzas entre ambos géneros (Figs. 15, 22) como son la presencia de antenas cortas, sólo algo más largas que las piezas bucales y distribución general de la quetotaxia cefálica y tegumentaria. Sin embargo en las larvas neonatas de Nomerobius casi todas las sedas dorsales del tórax son estrelladas en su extremo y en las de Sympherobius no, y sólo algunas están levemente espatuladas y hendidas en su extremo (Figs. 12, 19). En las larvas desarrolladas de Nomerobius las mandíbulas y maxilas son más estrechas, el primer artejo antenal no es fusiforme, sino cilíndrico, su extremo antenal carece de seta distal diferenciada, el número aparente de estemas es de 4 y no 3 , las setas pre- distales bien desarrolladas de la cápsula cefálica son 4 y no 3 , la zona externa de las maxilas porta un mayor número de setas, parece faltar la seta basal externa en el segundo segmento de los palpos y su último palpómero labial posee el área membranosa pequeña y elíptica en Nomerobius y grande, fusiforme y muy alargada en Sympherobius y la quetotaxia dorsal de los segmentos abdominales porta una robusta seta a cada lado ante los estigmas y no una mucho más pequeña y mucho más próxima a la línea media, diferencias a las que habría que añadir su coloración marcadamente disruptiva.

Anotamos cierta curiosa convergencia en el comportamiento corticícola y la presencia de pigmentación disruptiva de las larvas de Nomerobius y las de Brinckochrysa (Chrysopidae) (Tsukaguchi, 1977, 1979; Hölzel \& Duelli, 1990, 1994; Díaz Aranda \& Monserrat, 1992, etc.).

\section{Nomerobius spinosus Oswald, 1990}

Especie conocida de Chile y Argentina de biología casi desconocida.

MATERIAL ESTUdiado: 46: 2 o" or capturados a la luz.

Estos ejemplares han sido capturados a la luz, por lo que poco podemos aportar a su biología. En la línea de lo anotado por Monserrat (1998) las membranas peritróficas de alguno de los imagos estudiados portaban abundante material silíceo, también restos de pequeños artrópodos, hifas de hongos, esporas y polen diverso.

\section{Sympherobius gayi Navás, 1910}

Especie conocida de Argentina, Uruguay, Paraguay, Brasil, Chile, Perú e Isla de Pascua, que ha sido introducida en Nigeria como agente de control y, probablemente de forma accidental, en Portugal.

Material estudiado: 46: 2 \% . 53: 28. XII. 20019 o" o", 11. II. 200210 O , 17. II. 20023 O" O" (MACN), 7. III. 2002 2 ơ ơ, 1 \%, 8. III. 20021 \%, 10. III. 20021 on, 1 \%, 11. III. 20021 on, 1 q, 12. III. 20021 \% ex ovo, 14. III. 20021 \&, 23. III. 20024 ㅇ , 2. IV. 20025 ○゙ ○゙, 2 ๆ , 7. IV. 20021 o", 1 ᄋ, 1. VIII. $20021 \%$.

La biología de esta especie es casi desconocida y poco aportamos al respecto ya que todos los ejemplares aquí citados se recolectaron a la luz. En la línea de lo anotado por Monserrat (1998) las membranas peritróficas de alguno de estos ejemplares portaban abundantes restos de material silíceo diverso y numerosas hifas de hongos, también polen, esporas y restos de insectos muy triturados, particularmente áfidos y aleiródidos.

Algunos adultos que fueron mantenidos en cajas de cultivo se mantuvieron vivos 10, 24, 24, 32, 34, $35,37,42$ y 42 días según los ejemplares, observándose una marcada actividad en la búsqueda de alimento, especialmente en las horas del crepúsculo y durante la noche.

Los estadios preimaginales de esta especie son desconocidos y a partir de algunos ejemplares recolectados en la localidad 53 se obtuvieron varias puestas que han permitido completar su desarrollo preimaginal y describir sus fases larvarias.

Las hembras realizaban las puestas de forma intermitente y continuada a lo largo del tiempo en el que estuvieron vivas y no de una vez. Las puestas se practicaban durante la noche, especialmente sobre o cerca del margen de las hojas que contenían las colonias de áfidos utilizados para alimentar a los imagos. Los huevos quedaban adheridos longitudinalmente y de forma aislada. El número total de huevos puestos por cada hembra no pudo precisarse en la mayoría de las ocasiones, ya que habitualmente una misma caja de cultivo contenía varios ejemplares para inducir y favorecer la cópula y por otra parte la diferenciación de sexos es muy difícil de visu en ejemplares vivos, pero los datos anotados de hembras aisladas dieron unos resultados de 

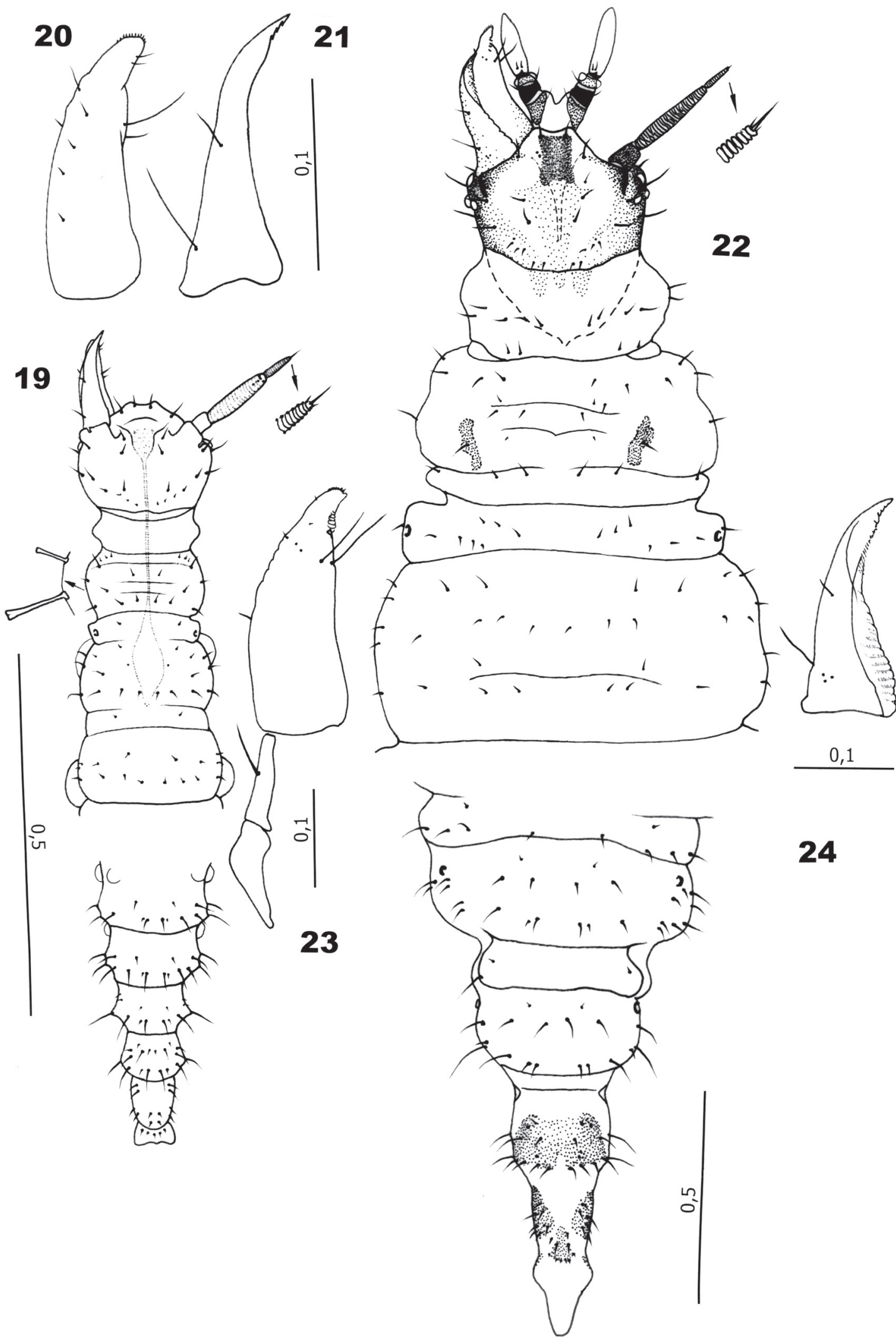
$5,6,22,23,23,25$ y 28 huevos. La mayoría de los huevos prosperaron, algunos no, probablemente por no estar fecundados, y se secaron a pesar del algodón humedecido.

Los huevos son brillantes, de color amarillento $\mathrm{y}$ tornan a pardo pálido conforme progresa su desarrollo. El micropilo es esférico, poco prominente y la superficie del corion que porta tenues impresiones foliculares. Completado su desarrollo embrionario practican una pequeña fisura longitudinal desde el micropilo. El tiempo para el desarrollo embrionario no pudo precisarse en la mayoría de las ocasiones, ya que habitualmente en una misma caja de cultivo se solapaban sucesivas puestas realizadas por una o varias hembras, pero los datos anotados de huevos aislados dieron unos resultados de 7-8 días.

Las larvas neonatas permanecen sobre el corion un tiempo antes de iniciar su actividad. Son muy activas e huidizas y tienden a esconderse en repliegues de las hojas o bajo ellas, por lo que son de muy difícil localización. Utilizan el extremo del abdomen como apoyo-palanca al caminar. Fueron alimentadas con ninfas e imagos de Brachycaudus (Appelia) schwartzi (Börner, 1931) y Aphis (Aphis) gossypii gossypii Glover, 1877. Mientras comen arquean las antenas hacia atrás y tras la ingesta dedican unos minutos al aseo, principalmente de las antenas. Realizan la primera muda a los 4-6 días y según los datos anotados completan su desarrollo larvario en 24, 28, 29 y 30 días desde la eclosión del huevo, tras los cuales inician la fabricación del capullo.

Las larvas neonatas (Figs. 19-21) son rosáceas muy pálidas con la cabeza levemente parda, especialmente en las zonas laterales. Las antenas, palpos y patas son pálidos. En la sutura frontal se ubican hileras de microtriquias y ventralmente parece desembocar una formación glandular, probablemente digestiva que alcanza el mesotórax. Algunas setas dorsales del tórax están levemente espatuladas y hendidas en su extremo.

Las larvas desarrolladas (Figs. 22-24, 37) son pardo-glauco pruinosas con dos bandas tenues pardas longitudinales sobre el dorso y lateralmente a ellas se disponen estrías blancuzcas en cada seg- mento. Setas dorsales pardo pálidas. Cápsula cefálica parcialmente embutida en el pronoto, está especialmente esclerificada y pigmentada de pardo en las zonas laterales, incluyendo los estemata, los escapos, la base de las piezas bucales y el segundo segmento de los palpos, en su zona dorsal se dispone una mancha longitudinal sobre la línea dorsal anterior media. Aparentemente un estema oscuro anterior y dos algo menores y posteriores. Mandíbulas, maxilas, palpos y antenas pardos. Pronoto con dos pequeños escleritos uno a cada lado. Patas pardo pálidas, más oscuras la mitad basal de las coxas y de los fémures. Quetotaxia, pigmentación estructural tegumentaria y morfología general según figuras 22-24.

Discusión: A pesar de tratarse de un género ampliamente distribuido en casi todos los continentes (salvo Australia y zona tropical del S. E. de Asia) y con más de 60 especies descritas y válidas, sólo existen datos de estadios preimaginales de nueve especies pertenecientes al género Sympherobius Banks, 1904. Ningún dato al respecto en las especies neotropicales y mayoritariamente pertenecen a especies frecuentes de las Regiones Paleártica y Neártica (Oswald, 1993a y Oswald \& Tauber, 2001).

La mayoría de las descripciones son casi testimoniales y no puede, por el momento, realizarse comparaciones en detalle respecto a la larva recién descrita, especialmente en lo que se refiere a la quetotaxia. El aspecto del huevo y las proporciones relativas y morfología general de la larva se mantienen y el patrón de coloración es similar entre la larva que hemos descrito y las larvas de este género descritas en la bibliografía (Essig, 1910; Smith, 1923, 1934; Withycombe, 1923, 1925; Bodenheimer, 1930; Killington, 1931, 1936, 1937; Silvestri, 1943; Nakahara, 1954; Hoffmann, 1962; New, 1967, 1968, etc.). Debe ponerse en duda la identidad de la larva descrita como perteneciente a S. matsucocciphagus Yang, 1980 por Yang (1980) cuyo patrón de coloración es diferente, como también lo es la longitud relativa de sus antenas respecto a todo lo conocido en el género Sympherobius, este hecho, ya anotado por Oswald \& Tauber

Figs. 19-24.- Sympherobius gayi. 19: Quetotaxia dorsal de la cabeza, tórax y extremo abdominal de la larva neonata, ampliadas dos setas y extremo antenal. 20: Maxila, dorsal. 21: Mandíbula, dorsal. 22: Quetotaxia dorsal de la cabeza, tórax y extremo abdominal de la larva desarrollada, ampliado extremo antenal. 23: Maxila, dorsal. 24: Mandíbula, dorsal. Escala en mm.

Figs. 19-24.- Sympherobius gayi. 19: Dorsal chaetotaxy of head, thorax and abdominal tip of the neonate larva, magnified two setae and antenal tip. 20: Maxilla, dorsal. 21: Mandible, dorsal. 22: Dorsal chaetotaxy of head, thorax and abdominal tip of the full grown larva, magnified antenal tip. 23: Maxilla, dorsal. 24: Mandible, dorsal. Scale in $\mathrm{mm}$. 
(2001), hace cuestionable su correcta asignación a este género. También la puesta cerca del borde de las hojas había sido citada por Withycombe, 1923 o Killington, 1936, 1937 para S. elegans (Sthephens, 1836) y S. pygmaeus (Rambur, 1842).

\section{Megalominae Krüger, 1922}

\section{Megalomus stangei González Olazo, 1981}

Especie de biología muy poco conocida y citada en zonas de influencia andina de Chile y Argentina.

Material estudiado: 24: 1 o capturado a la luz. 33: 1 \% sobre Nothofagus nitida (Phil.).

Esta especie ofrece una gran variabilidad en la pigmentación de sus alas (Monserrat, 1997), las figuras 38 y 39 añaden nuevos datos al respecto. Las membranas peritróficas de estos ejemplares portaban abundantes restos de áfidos, escamas de lepidópteros, hifas de hongos, polen y material silíceo diverso.

\section{Drepanepteryginae Krüger, 1922}

Gayomyia falcata (Blanchard en Gay, 1851)

Especie de biología poco conocida y citada de Argentina, Chile, Archipiélago de Juan Fernández y Bolivia.

Material estudiado: $14: 2$ \% . $24: 1$. $32: 1$. 34 : 1 ○’, 1 우 ㅇ․ . 50: 3 ㅇ ○”, 1 우.

De ellos 6 se han recolectado a la luz, 2 sobre Nothofagus nitida (Phil.), 1 sobre Nothofagus betuloides (Mirb.) y 1 sobre Laurelia sempervirens (Ruíz \& Pavón).

Como en la especie anterior, esta especie ofrece una gran variabilidad en la pigmentación de sus alas (Monserrat, 1998). La figura 40 añade nuevos datos al respecto. En la línea de lo anotado por Monserrat (1998) las membranas peritróficas de estos ejemplares portaban abundantes cantidades de restos de áfidos. Algunos adultos fueron conservados en cajas de cultivo se mantuvieron vivos 28 , 29 y 41 días, observándose una escasa, casi nula, actividad y permaneciendo durante horas, tras abrir la tapa de la caja que los contenía, en estado quiescente de tanatosis con la cabeza y las antenas replegadas entre el campo costal de sus alas anteriores y simulando una hoja seca con sus crípticas alas.

Los estadios preimaginales de este género / especie son desconocidos (Oswald, 1993a; Oswald \& Tauber, 2001) y sólo Monserrat (1998) aporta algunos datos sobre el huevo, la puesta y la biolo- gía de los neonatos de esta especie, anotando una puesta realizada durante la noche formada por dos grupos de 3 y 2 huevos alargados, verticalmente adheridos, de color blanco al inicio y verdosos-parduscos al avanzar su desarrollo que duró 6 días y cuyas neonatas sobrevivieron 6 días sin optar por comer las ninfas de áfidos que en su momento se les proporcionaron.

A partir de algunos de los ejemplares recolectados en las localidades 14, 32 y 50 se obtuvieron varias puestas que han permitido completar su desarrollo preimaginal y describir sus fases larvarias.

Las hembras realizaron las puestas de forma intermitente y continuada a lo largo del tiempo en el que estuvieron vivas y no de una vez. Las puestas se practicaban durante la noche sobre la superficie de las hojas que contenían las colonias de áfidos utilizados para alimentar a los imagos o en las fibras de nylon que se utilizaba como respiradero de las cajas de cultivo que los contenían. Los huevos quedaban adheridos verticalmente, aislados o en grupos de $1,2,3,3,4,5,5,6,7,8$ y 8 huevos, de forma similar a lo anotado por Fulmek (1941) o Gepp (1990, 1999) para Drepanepteryx phalaenoides (L.) especie Paleártica de la misma subfamilia. El número total de huevos puestos por cada hembra fue de 1, 5, 21 y 25 . La mayoría de los huevos no prosperaron, probablemente por no estar fecundados, y se secaron a pesar del algodón humedecido. Los huevos son de color blancuzco y tornan a pardo conforme progresa su desarrollo. El micropilo es prominente y fungiforme, la superficie del corion porta impresiones foliculares cilíndricas de contorno irregular. La eclosión de los huevos viables se produce entre los 6 y los 7 días de su puesta mediante una fisura longitudinal que desde el micropilo abarca un tercio de la longitud del huevo.

Las larvas neonatas permanecen sobre el corion un tiempo antes de iniciar su actividad. Son muy huidizas y tienden a esconderse en los repliegues de las hojas o bajo ellas, por lo que son de muy difícil localización. Utilizan el extremo del abdomen como apoyo-palanca al caminar. Fueron alimentadas con ninfas e imagos de Brachycaudus (Appelia) schwartzi (Börner, 1931) y Aphis (Aphis) gossypii gossypii Glover, 1877. Mientras comen arquean las antenas hacia atrás y después dedican unos minutos al aseo, principalmente de las antenas. Realizan la primera muda a los 3-4 días y completan su desarrollo en unos 15-16 días desde la eclosión del huevo, tras los cuales inician la fabricación del capullo.

Las larvas neonatas (Figs. 25-27) son de tegumento pardo muy pálido, en este estadio sus setas son levemente espatuladas y de color negro, poco 


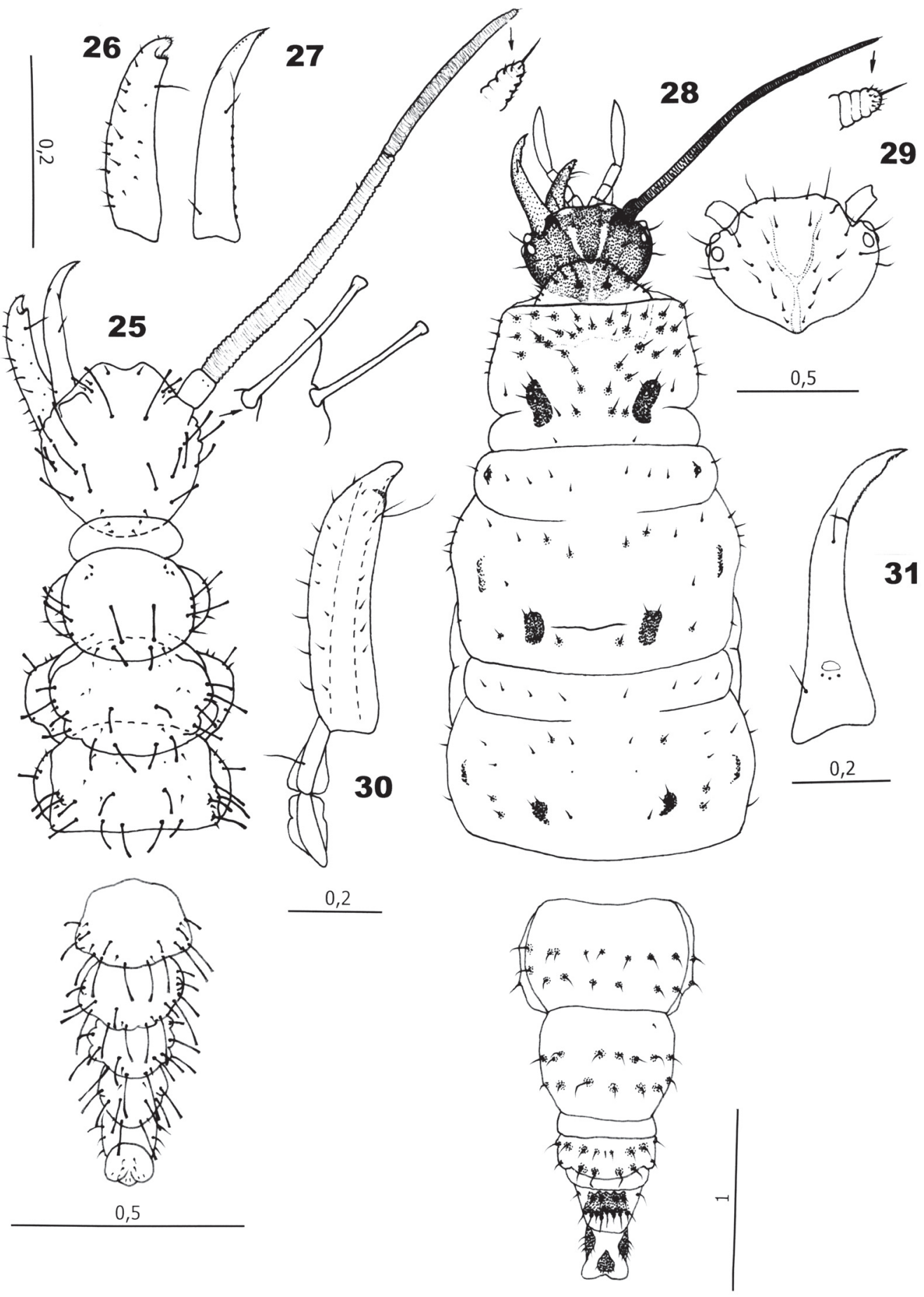

Figs. 25-31.- Gayomyia falcata. 25: Quetotaxia dorsal de la cabeza, tórax y extremo abdominal de la larva neonata, ampliadas dos setas y extremo antenal. 26: Maxila, dorsal. 27: Mandíbula, dorsal. 28: Quetotaxia dorsal de la cabeza, tórax y extremo abdominal de la larva desarrollada, ampliado extremo antenal. 29: Cápsula cefálica, dorsal. 30: Maxila, dorsal. 31: Mandíbula, dorsal. Escala en mm.

Figs. 25-31.- Gayomyia falcata. 25: Dorsal chaetotaxy of head, thorax and abdominal tip of the neonate larva, magnified two setae and antenal tip. 26: Maxilla, dorsal. 27: Mandible, dorsal. 28: Dorsal chaetotaxy of head, thorax and abdominal tip of the full grown larva, magnified antenal tip. 29: Cephalic capsule, dorsal. 30: Maxilla, dorsal. 31: Mandible, dorsal. Scale in mm. 

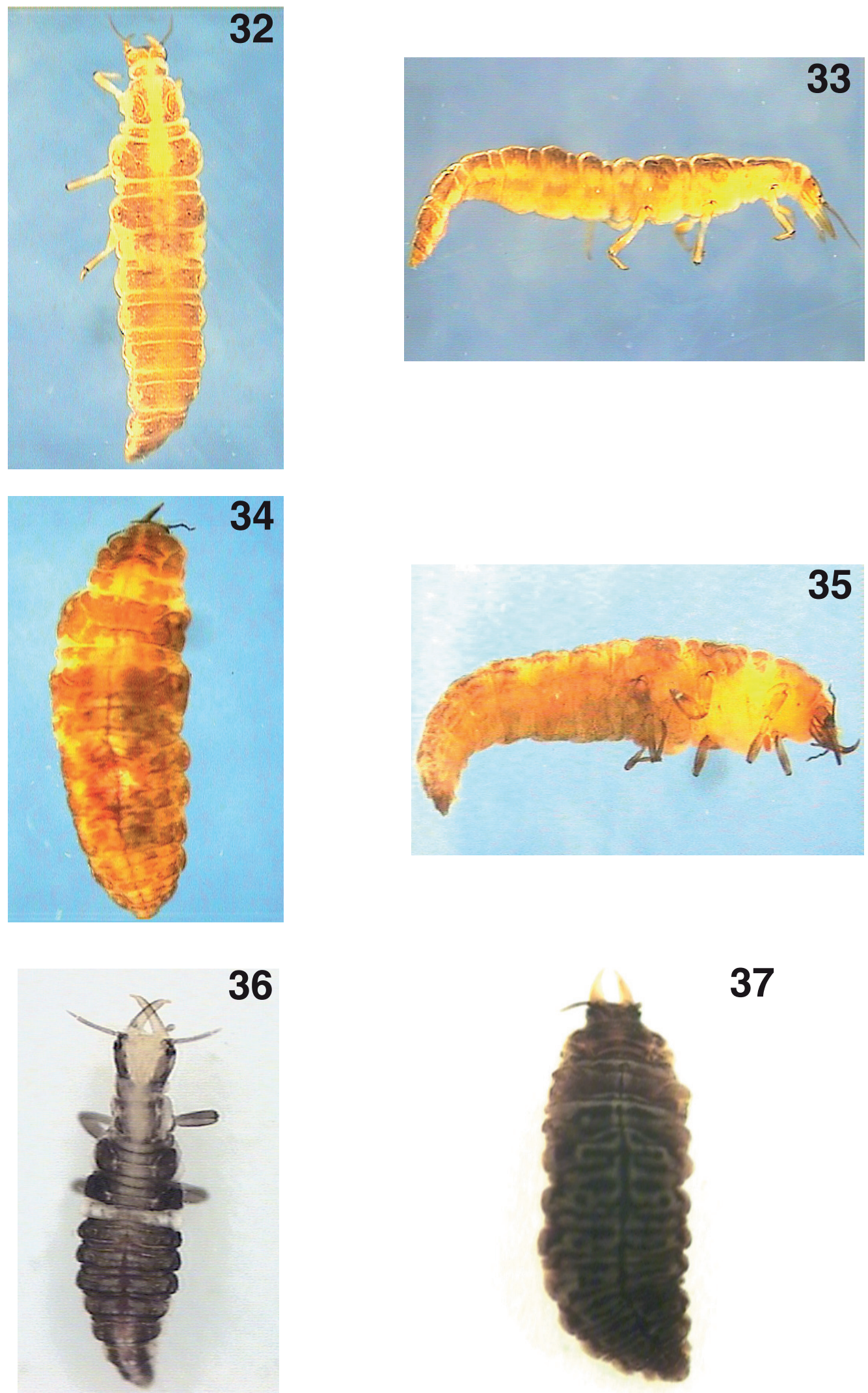

37

(c) Sociedad de Amigos del Museo

Nacional de Ciencias Naturales y

Consejo Superior de Investigaciones Científicas Licencia Creative Commons 3.0 España (by-nc) 
tiempo después tornan a más rojizas y esclerificadas con cabeza más oscura lateralmente y con antenas y palpos blancuzcos. Estemas negros. Poseen una tenue línea media dorsal blancuzca con una banda pardo- rojiza longitudinal a cada lado, desde la zona lateral de la cabeza al extremo del abdomen donde son algo más oscuras. Estas bandas forman tenues estrías trasversales más oscuras entre los segmentos, principalmente en los del tórax y son más anchas y oscuras en el pronoto. Quetotaxia tegumentaria y morfología general según figuras 25-27.

Las larvas desarrolladas (Figs. 28-31) son más pardas, menos rojizas, con setas dorsales pardo pálidas y cuya base tegumentaria está generalmente más esclerificada y pigmentada afectando al tegumento circundante, especialmente en el pronoto y segmentos terminales del abdomen. Cápsula cefálica relativamente poco esclerificada, pigmentada de pardo dorsalmente, más parda y esclerificada en las zonas laterales, incluyendo los estemas, los escapos y la base de las piezas bucales, en su zona dorsal se limita una pequeña lengüeta que se dispone sobre la línea dorsal media y está limitada por una sutura en Y más pálida. Aparentemente tres pequeños estemas oscuros sobre la base de las antenas y uno algo mayor más posterior. Mandíbulas, maxilas, palpos y antenas pardo pálidos. Palpos labiales con último palpómero subcilíndrico y zona membranosa ventral muy alargada y penúltimo palpómero el doble de largo que de ancho. Pronoto con una banda media blancuzca y una banda pardo rojiza arqueada a cada lado. Desde el mesonoto hasta el final del abdomen existe dorsalmente una banda longitudinal media de color pardo flanqueada a cada lado por una banda blancuzca, levemente convexa y arqueada hacia el final del abdomen donde convergen la de uno y otro lado conforme se hacen más tenues y fraccionadas y existe una segunda banda, más externa, lateral y más oscura y ancha, que es particularmente patente en el metatórax. Patas pardo pálidas, más oscuros los fémures, tercio basal de las tibias y ápice distal de los tarsos.

Figs. 32-37. - Larva desarrollada de: 32 Hemerobius bolivari, dorsal. 33 Hemerobius bolivari, lateral. 34 Hemerobius chilensis, dorsal. 35 Hemerobius chilensis, lateral. 36 Larva de segundo estadio de Nomerobius cuspidatus, dorsal. 37 Larva desarrollada de Sympherobius gayi, dorsal.

Figs. 32-37.- Full grown larva of: 32 Hemerobius bolivari, dorsal. 33 Hemerobius bolivari, lateral. 34 Hemerobius chilensis, dorsal. 35 Hemerobius chilensis, lateral. 36 Second instar larva of Nomerobius cuspidatus, dorsal. 37 Full grown larva of Sympherobius gayi, dorsal.
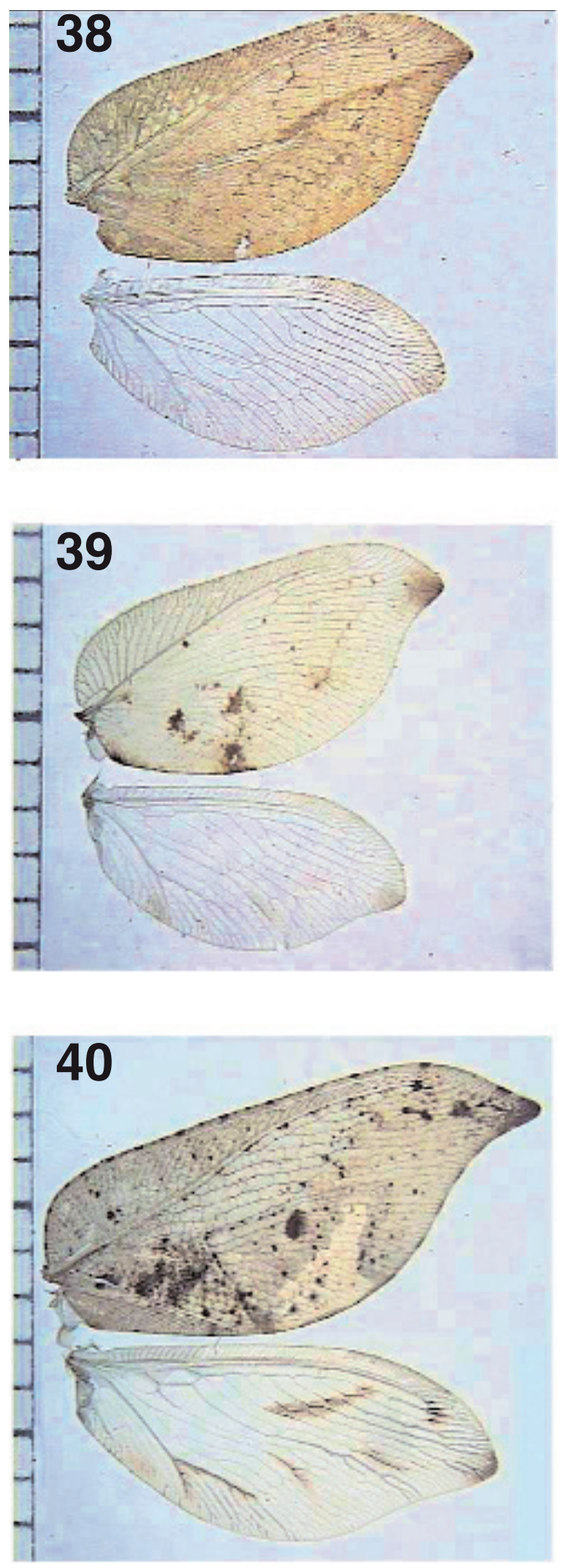

Figs. 38-40.- Alas de: 38 Megalomus stangei, de la localidad 24. 39 Megalomus stangei, de la localidad 33. 40 Gayomyia falcata, de la localidad 32. Escala en mm.

Figs. 38-40.- Wings of: 38 Megalomus stangei, from locality 24. 39 Megalomus stangei, from locality 33. 40 Gayomyia falcata, from locality 32 . Scale in $\mathrm{mm}$. 
Quetotaxia, pigmentación estructural tegumentaria y morfología general según figuras 28-31.

Discusión: Los estadios larvarios del género Gayomyia eran desconocidos (Oswald, 1993a; Oswald \& Tauber, 2001) y dentro de la subfamilia Drepanepteryginae Krüger, 1922, a la cual pertenece, sólo existen datos del género Drepanepteryx, en particular de la especie Paleártica $D$. phalaenoides (Linnaeus, 1758). Las referencias existentes en la bibliografía sobre los estadios larvarios de esta especie son, en general, muy antiguos, poco precisos o corresponden a fotografías macroscópicas de ejemplares y poseen escasos elementos que permitan conocer detalles sobre su quetotaxia, especialmente en las larvas desarrolladas (Réaumur, 1737; Von Gleichen, 1770; Evans, 1848; Brauer, 1867; Standfuss, 1906, 1910; Morton, 1910; Killington, 1936, 1937; Fulmek, 1941; Christensen, 1943; Hoffman, 1962; Gepp, 1986a, b, 1999). En cualquier caso pueden deducirse semejanzas entre la puesta, la morfología de los huevos y las larvas de ambos géneros como son la presencia de sutura en Y, lengüeta dorsal cefálica, débil esclerificación de la región anterior de la cápsula cefálica, longitud relativa de las antenas respecto a las piezas bucales, escasa complejidad de sus procesos apicales, presencia de escleritos torácicos y setas con esclerificación basal. Parece, no obstante, diferir en la longitud relativa de los dos segmentos del flagelo (similar en $D$. phalaenoides y bastante mayor el $1^{\circ}$ respecto al $2^{\circ}$ en $G$. falcata) y en la disposición y morfología de las setas, al menos en las larvas neotatas (ver Fulmek, 1941). En todo caso, la controversia existente sobre la morfología de esta larva y en particular sobre su posible fisiogastria supuestamente anotada por MacLeod, 1960 (según indican Oswald \& Tauber, 2001) debe disiparse, ya que MacLeod, 1960 se refiere a Boriomya fidelis (Banks, 1897), hoy día Megalomus fidelis (Banks, 1897), y no a Drepanepteryx.

\section{Conclusiones}

Debido a las condiciones climatológicas tan particulares de esta extensa zona meridional de la Región Neotropical, especialmente debido a la presencia de fuertes y persistentes vientos, a la existencia de un relativamente corto periodo de bonanza primaveral-estival con duras condiciones invernales y a la pobreza y uniformidad en sus medios, sólo más diversificados en las zonas de influencia andina y pacífica, cabría esperar pocos resultados tras unos muestreos en los que se trataba de localizar un grupo tan termófilo y tan sensible al viento, especialmente habiéndose utilizando el tipo de muestreo mediante mangueo de la vegetación anteriormente citado. Aun así, se han hallado 10 especies de hemeróbidos capturadas en 48 localidades de Patagonia y Tierra del Fuego, en algunos casos con poblaciones aparentemente muy numerosas.

Algunas especies como Hemerobius bolivari parecen ser extremadamente oportunistas y colonizan un gran número de medios, latitudes y altitudes, por el contrario, otras especies como Hemerobius chilensis, circunscrita a bosques de Nothofagus, Nomerobius signatus aparentemente asociada a Austrocedrus chilensis (Don.) Florin et Boutleje o Hemerobius stenopterus limitada al húmedo sotobosque valdiviano, parecen ser extremadamente estenoicas en la elección de su planta substrato o de su medio.

A diferencia con otras familias de Neurópteros, y según los datos existentes en la bibliografía y a los ahora obtenidos, el límite meridional en la distribución de los Hemeróbidos en Sudamérica alcanza el propio límite del continente, habiéndose capturado ejemplares en el extremo más meridional del mismo, donde las condiciones climatológicas son especialmente severas.

\section{AGRADECIMIENTOS}

Deseo manifestar mi agradecimiento a Flavio $\mathrm{N}$. Moschione y a Victoria Massola del Departamento de Áreas protegidas de la Dirección Provincial de Recursos Naturales de la Provincia de Buenos Aires y a Alejandra Silva, Arturo Rosas e Ivan Benoit del Departamento de Patrimonio Silvestre del CONAF por los permisos de muestreos en áreas protegidas que me fueron concedidos. A Alicia Miravalle del Departamento de Biología, Bioquímica y Farmacia y a Rodrigo Tizón del Departamento de Botánica de la Universidad del Sur en Bahía Blanca por su apoyo y por su ayuda en la identificación de algunas de las plantas aquí citadas, a Juan M. Nieto Nafría de la Universidad de León por identificarme los pulgones que se citan, a Eduardo Ruiz por su ayuda en el tratamiento de las imágenes y a Enrique Garavano por acompañarme con su mate y su paciencia durante los muestreos.

\section{Referencias}

AsPöck, H., Aspöck, U. \& Hölzel, H., 1980. Die Neuropteren Europas. Goecke \& Evers. Krefeld. t. I, 495 pp., t. II, 355 pp.

Bodenheimer, F. S., 1930. Sympherobius (Nefasitus) amicus Nav. In Die Schädlings fauna Palästinas. Monographien zur angewndten Entomologia, 10: 137-140. 
Brauer, F., 1867. Beitrag zur Kenntniss der MantispidenGattungen. Verhandlungen der zoologischen- botanischen Gesellschaft in Wien, 17: 281-286.

Christensen, P. J. H., 1943. Om morphologien og biologien hos Drepanepteryx phalaenoides I. Flora og Fauna, 49: 1-13.

Díaz Aranda, L. M. \& Monserrat, V. J., 1992. Descripción de los estadios larvarios de Brinckochrysa nachoi Monserrat, 1977 (Neuroptera, Chrysopidae). Nouvelle Revue d'Entomologie ( $N$. S.), 9(3): 207-214.

Erize, F., 2000a. Especies forestales de la Argentina occidental I. El Ateneo. Buenos Aires. 120 pp.

ERIzE, F., 2000b. Especies forestales de la Argentina oriental II. El Ateneo. Buenos Aires. 124 pp.

Essig, F. O., 1910. The Natural enemies of the Citrus mealy Bug. I. Pomona College Journal of Entomology, II: 143-146.

Evans, W. F., 1848. Monograph of the British Species of the genus Chrysopa. Transactions of the Entomological Society of London, 5: 77-79, pl. IX-X.

FulmeK, L., 1941. Über die Aufzucht von Drepanepteryx phalaenoides L. ex ovo. (Neuroptera: Planipennia, Hemerobiidae). Arbeiten über morphologische und taxonomische Entomologie aus BerlinDahlem, 8: 127-130.

GepP, J., 1986a. Die Netzflügler eine faunistische Übersicht. Naturkundliche Forschung im Fürstentum Liechthenstein, 6: 1-26.

GEPP, J., 1986b. Biology and Larval Diagnosis of Central European Neuroptera (A Review of Present Knowledge). In: J. Gepp, H. Aspöck \& H. Hölzel (eds.). Recent Research in Neuropterology. Graz: 137-144.

GEPP, J., 1990. An illustrated review of egg morphology in the families of Neuroptera (Insecta: Neuropteroidea). In: M. W. Mansell \& H. Aspöck, (eds.). Advances in Neuropterology. Proceedings of the Third International Symposium on Neuropterology. Pretoria: 131-149.

GePP, J., 1999. Neuropteren als Indikatoren der Naturraubewertung. In: H. Aspöck, (ed.). Neuropterida: Raphidioptera, Megaloptera, Neuroptera. Kamelhälse, Schlammfliegen, Ameisenlöwen. Biologiezentrum des Oberösterreich Landesmuseum, 138: 167-208.

GonzÁlez Olazo, E. V., 1992. Una nueva especie de Nomerobius (Neuroptera, Hemerobiidae) de la Argentina. Acta Zoológica Lilloana, 42(1): 109-111.

GonzÁlez Olazo, E. V., 1993. Contribución al conocimiento de las especies de Nusalala (Neuroptera, Planipennia, Hemerobiidae) de la Argentina. Acta Zoológica Lilloana, 42(2): 215-224.

GonzÁlez Olazo, E. V., 1996. Catálogo de los tipos de Neuroptera (Insecta: Holometabola) depositados en colecciones argentinas. Acta Zoológica Lilloana, 43(2): 373-391.
Haene, E. \& Gostaro, A., 2001. 100 árboles argentinos. Albatros. Buenos Aires. 189 pp.

Hoffman, J., 1962. Faune des Nevroptéroïdes du GrandDuché de Luxembourg. Archives de l'Institut Grandducal de Luxembourg (N. S.), 28: 249-332.

Hoffmann, A. E., 1997. Flora silvestre de Chile. Fundación Claudio Gay. Santiago. 253 pp.

Hölzel, H. \& Duelli, P., 1990. Remarks on the Chrysopidae of Madagascar (Insecta: Neuroptera). In: M. Mansell \& H. Aspöck (eds.). Advances in Neuropterology. Proceedings of the Third International Symposium on Neuropterology. Pretoria: 271-275.

Hölzel, H. \& Duelli, P., 1994. Brinckochrysa beninensis n. sp.- eine neue Chrysopiden- Spezies aus Westafrika (Neuroptera: Chrysopidae). Entomologische Zeitschrift, 104(3): 54-58.

Killington, F. J., 1931. Notes on the Life- History of Sympherobius fuscescens Wall. (= inconspicuous McL.) (Neuroptera). The Entomologist, 64: 217-223.

Killington, F. J., 1936. A monograph of the British Neuroptera I. Ray Society. London. 269 pp., pl. I-XV.

Killington, F. J., 1937. A monograph of the British Neuroptera II. Ray Society. London. 306 pp., pl. XVI-XXX.

McEwen, P., New, T. R. \& Whittington, A. E., 2001. Lacewings in the Crop Environment. Cambridge University Press. Cambridge. 546 pp.

MAcLeod, E. G., 1960. The Immature Stages of Boriomyia fidelis (Banks) with Taxonomic Notes on the Affinities of the Genus Boriomyia (Neuroptera: Hemerobiidae). Psyche, 67: 26-40, 99.

Monserrat, V. J., 1996. Revisión del género Hemerobius de Latinoamérica (Neuroptera, Hemerobiidae). Fragmenta entomologica, 27(2): 399-523.

Monserrat, V. J., 1997. Revisión del género Megalomus de Latinoamérica (Neuroptera, Hemerobiidae). Fragmenta entomologica, 29(1): 123-206.

Monserrat, V. J., 1998. Nuevos datos sobre los hemeróbidos de América (Neuroptera: Hemerobiidae). Journal of Neuropterology, 1: 109-153.

Monserrat, V. J., 2000. Revisión del género Nusalala (Neuroptera, Hemerobiidae). Fragmenta entomologica, 32(1): 83-162.

Monserrat, V. J. \& Deretsky, Z., 1999. New Faunistical, Taxonomic and Systematic Data on Brown Lecewings (Neuroptera, Hemerobiidae). Journal of Neuropterology, 2: 45-66.

Morton, K. J., 1910a. Life- History of Drepanopteryx phalaenoides, Linn. The Entomologist Monthly Magazine, 46: 54-62, pl. II.

NAKAHARA, W., 1954. Early stages of some Japanese Hemerobiidae, including two new species. Konty $\hat{u}$, 21: 41-46, pl. II-VI. 
NEw, T. R., 1967. Biological notes on Sympherobius pellucidus (Walk.), with a description of the larva (Neuroptera: Hemerobiidae). Entomologist's Gazette, 18: $50-52$

NEw, T. R., 1968. Sympherobius pygmaeus (Ramb.) (Neur., Hemerobiidae) in Berkshire, with a description of the cocoon and pupa. The Entomologist Monthly Magazine, [1967], 103: 171-172.

Oswald, J. D., 1990. Revision of the Neotropical Brownlacewing Genus Nomerobius (Neuroptera, Hemerobiidae). Annals of the Entomological Society of America, 83(1): 18-29.

Oswald, J. D., 1993a. Revision and cladistic analysis of the world genera of the family Hemerobiidae (Insecta: Neuroptera). Journal of the New York Entomological Society, 101(2): 143-299.

Oswald, J. D., 1993b. A new genus and species of brown lacewing from Venezuela (Neuroptera: Hemerobiidae), with comments on the evolution of the hemerobiid forewing radial vein. Systematic Entomology, 18: 363-370.

OswaLD, J. D., 1994. A new phylogenetically basal subfamily of brown lacewings from Chile (Neuroptera, Hemerobiidae). Entomologica Scandinavica, 25: 295-302.

Oswald, J. D. \& Tauber, C. A., 2001. Preimaginal stages of the family Hemerobiidae. In: P. McEwen, T. R. New \& A. E. Whittington. (eds.). Lacewings in the Crop Environment. Cambridge University Press. Cambridge: $50-60$.

Penny, N., 2002. A Guide to the Lacewings (Neuroptera) of Costa Rica. Proceedings of the California Academy of Sciences, 53(12): 161-457.

Penny, N. \& LeE, V. F., 1996. Types of Mecoptera, Megaloptera, Raphidioptera, and Neuroptera (Insecta) in the collection of the California Academy of Sciences. Proceedings of the California Academy of Sciences, 49(4): 127-149.

Perez Gelabert, D. E. \& Flint, O. S., 2001. Annotated List of the Neuroptera of Hispaniola, with New Faunnistic Records of some species. Journal of Neuropterology, 3: 9-23.

RÉAumur, R. A. F., 1737. Mémoires pour servir à l'histoire des insectes, vol. 3, Neuropterida, Paris: 363-412. (no visto).

Silvestri, F., 1943. Compendio di Entomologia applicata (Agraria-Forestale-Medica - Veterinaria), vol. II. Portici. 512 pp., Ordo Neuroptera: 1-33.

SMith, R. C., 1923. The life histories and stages of some Hemerobiids and allied species. Annals of the Entomological Society of America, 16: 129-151.
Smith, R. C., 1934. Notes on the Neuroptera and Mecoptera of Kansas, with keys for the identification of species. Journal of the Kansas Entomological Society, 7: 120-145.

STANDFuss, M., 1906. Bewegliche Puppen bei Insekten mit vollkommener Verwandlung. Mitteilungen der schweizerischen entomologischen Gesellschaft,11: 154.

STANDFuss, M., 1910. Notes on the Biology of Drepanepteryx phalaenoides L. The Entomologist Monthly Magazine, 46: 60-62.

TJeder, B., 1961. Neuroptera- Planipennia. The lacewings of Southern Africa 4. Family Hemerobiidae. In: B. Hanström, P. Brinck. \& G. Rudebec (eds.). South African Animal Life. Swedish Natural Science Research Council. Stockholm, 8: 296-408.

Tsukaguchi, S., 1977. [Biology and rearing of Green Lacewings.] Insectarium, 1977(14): 180-184 (en japonés).

TsukAGUCHI, S., 1979. Taxonomic Notes on Brinckochrysa kintoki (Okamoto) (Neuroptera: Chrysopidae). Kontyû, 47(3): 358-366.

Von Gleichen, W. F. F., 1770. Versucheiner Geschichte der Blatläuse und Blatlausfresser des Ulmenbaums, Nüremberg. (no visto).

Withycombe, C. L., 1923. Notes on the biology of some British neuroptera (Planipennia). Transactions of the Entomological Society of London, 1922: 501-594, pl. XXXVIII-XLIII.

Withycombe, C. L., 1925. Some aspects of the biology and morphology of the Neuroptera. With special reference to the immature stages and their possible phylogenetic significance. Transactions of the Entomological Society of London, 1924: 303-411, pl. XXXIX-XLIV.

YANG, C. K., 1980. Three new species of Sympherobius from China (Neuroptera: Hemerobiidae). Acta Agriculturae Universitatis Pekiensis, 6(1): 87-92. 